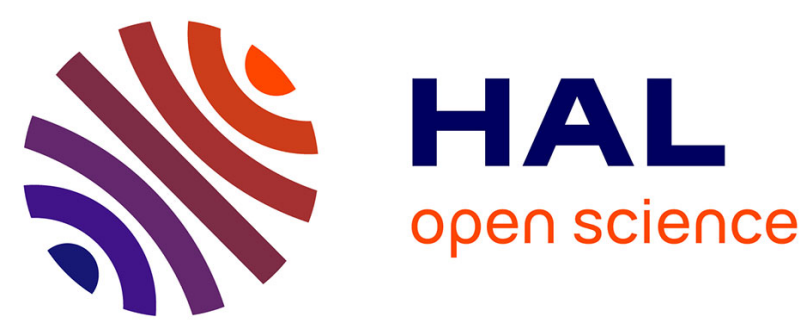

\title{
SFH2 regulates fatty acid synthase activity in the yeast Saccharomyces cerevisiae and is critical to prevent saturated fatty acid accumulation in response to haem and oleic acid depletion
}

Thomas Desfougères, Thierry Ferreira, Thierry Bergès, Matthieu Régnacq

\section{To cite this version:}

Thomas Desfougères, Thierry Ferreira, Thierry Bergès, Matthieu Régnacq. SFH2 regulates fatty acid synthase activity in the yeast Saccharomyces cerevisiae and is critical to prevent saturated fatty acid accumulation in response to haem and oleic acid depletion. Biochemical Journal, 2008, 409 (1), pp.299-309. 10.1042/BJ20071028 . hal-00478860

\section{HAL Id: hal-00478860 https://hal.science/hal-00478860}

Submitted on 30 Apr 2010

HAL is a multi-disciplinary open access archive for the deposit and dissemination of scientific research documents, whether they are published or not. The documents may come from teaching and research institutions in France or abroad, or from public or private research centers.
L'archive ouverte pluridisciplinaire HAL, est destinée au dépôt et à la diffusion de documents scientifiques de niveau recherche, publiés ou non, émanant des établissements d'enseignement et de recherche français ou étrangers, des laboratoires publics ou privés. 


\title{
SFH2 regulates fatty acid synthase activity in the yeast Saccharomyces cerevisiae and is critical to prevent saturated fatty acid accumulation in response to haem and oleic acid depletion
}

\author{
Thomas Desfougères, Thierry Ferreira, Thierry Bergès and Matthieu Régnacq* \\ Laboratoire de Génétique de la Levure CNRS-UMR6161, Université de Poitiers, 40 avenue du \\ Recteur Pineau, 86022 POITIERS CEDEX, France
}

Keywords: SEC14/ oleic acid/ fatty acid/anaerobiosis/ neutral lipids/ fatty acid synthase

Page heading title: Sfh2p inhibits yeast fatty acid synthase

Abbreviations used : DAG, Diacylglycerol; FAS, fatty acid synthase; PtdCho, Phosphatidyl choline; PtdEtn, Phosphatidyl ethanolamine; PtdIns, Phosphatidyl inositol; PtdOH, phosphatidic acid; PtdSer, Phosphatidyl serine; SFA, saturated fatty acids; TAG, Triacylglycerol; UFA, unsaturated fatty acids;

*To whom correspondance should be addressed : Laboratoire de Génétique de la Levure CNRSUMR6161, Université de Poitiers, 40 avenue du Recteur Pineau, 86022 POITIERS CEDEX, France. Phone: (33) 549453 864. Fax: (33) 549454186

E-mail: matthieu.regnacq@univ-poitiers.fr 


\begin{abstract}
The yeast Saccharomyces cerevisiae is a facultative anaerobic organism. In anaerobiosis, sustained growth relies on the presence of exogenously supplied unsaturated fatty acids and ergosterol that yeast is unable to synthesize in the absence of oxygen or upon haem depletion. In the absence of exogenous supplementation with unsaturated fatty acid, a net accumulation of saturated fatty acid (SFA) is observed that induces significant modification of phospholipid profile [1]. Herein, we focus on the role of SFH2/CSR1, a hypoxic gene related to SEC14 and its involvement in lipid metabolism upon haem depletion in the absence of oleic acid supplementation. We observed that inactivation of SFH2 results in enhanced accumulation of SFA and phospholipid metabolism alterations. It results in premature growth arrest and leads to an exacerbated sensitivity to exogenous SFA. This phenotype is suppressed in the presence of exogenous oleic acid, or by a controlled expression of FAS1 one of the two genes encoding FAS. We present several lines of evidence to suggest that $\mathrm{Sfh} 2 \mathrm{p}$ and oleic acid regulate SFA synthase in yeast at different levels: whereas oleic acid acts at the transcriptional level on FAS2, we show that Sfh2p inhibits fatty acid synthase activity in response to haem depletion.
\end{abstract}




\section{INTRODUCTION}

The budding yeast Saccharomyces cerevisiae is a facultative anaerobic organism, which can alternatively utilize respiration or fermentation for its energetic requirements. Physiological adaptation to aerobiosis and anaerobiosis is accompanied by the differential expression of a large number of genes in relation to oxygen availability [2-4]. Respiration deficiency resulting from oxygen limitation has several consequences, among which unsaturated fatty acids and ergosterol deprivation are potentially sufficient to compromise growth. Indeed, the biosynthetic pathways of these essential molecules are strictly aerobic. Transcriptional induction of hypoxic genes encoding oxygen dependent enzymes including ERG11 (for sterol biosynthesis) and OLE1 (for fatty acid desaturation) can be a temporary mean for the cells to counteract oxygen limitation. However, long term growth in strict anaerobiosis necessitates supplementation with ergosterol and oleic acid as a source of unsaturated fatty acid.

Since haem is a cofactor of several enzymes necessary for sterol and unsaturated fatty acid synthesis, a major consequence of haem depletion in yeast is unsaturated fatty acid and sterol biosynthesis preclusion. In addition, haem depletion also results in activation of hypoxic genes through its effect on several transcriptional factors including Rox1p and Hap 1p [2, 5]. Haem depletion can be easily achieved in a strain deleted for the $H E M 1$ gene, which encodes the $\delta$ aminolevulinic acid synthase gene. In the presence of exogenous $\delta$-aminolevulinic acid, a hem $1 \Delta$ strain is able to synthesize unsaturated fatty acid and ergosterol and hypoxic genes are repressed: this corresponds to aerobic-like growth. In contrast, a hem $1 \Delta$ mutant cultivated in complete medium lacking $\delta$-aminolevulinic acid can mimic most of anaerobiosis-induced effects of oxygen deprivation, including ergosterol and oleic acid requirement for robust growth, and hypoxic gene induction $[6,7]$.

In this experimental system, unsaturated fatty acid starvation in haem depleted medium results in growth arrest after 5 generations. Interestingly, cells accumulate saturated fatty acids (SFA) which are not mainly stored within triacylglycerols and steryl esters but rather within specific phospholipid species, with a marked preference for PtdIns [1]. In yeast, the balance of PtdCho versus PtdIns is controlled by Sec14p, an essential protein that is involved in protein transport from the yeast Golgi complex. More specifically, it is currently proposed that this protein regulates the interface between phospholipids and proteins that participate in the traffic machinery in the secretory pathway [8]. Numerous data suggest that Sec14p controls phosphatidylcholine 
(PtdCho) metabolism through the CDP-choline pathway. Indeed some of the mutants that relieve cells of Sec14p requirements (the so-called "by-pass Sec14p" mutants) inactivate structural genes for enzymes of the CDP-choline pathway (e.g., CKI1, CPT1) for PtdCho biosynthesis. In addition, biochemical data indicate that Sec14p can inhibit Pct1p, an enzyme of the CDP-choline pathway [9].

Overexpression of $\mathrm{SFH} 2$ was shown to suppress the thermosensitive phenotype of sec 14-1 [10]. Sfh2p belongs to a family of 5 yeast proteins which are structurally homologous to Sec14p, hence its name ( $\underline{\operatorname{Sec}} \underline{F}$ ourteen $\underline{H}$ omolog $\underline{2})$. All, but Sfh1p, exhibit PtdIns but not PtdCho-transfer activity. Some experimental evidences support a role for Sfh4p at contact sites between ER and Golgi membranes to facilitate PtdSer translocation. The phenotype of sfh $3 \Delta$ strain, and the localization of Sfh3p in lipid particle, led to propose that $S F H 3$ could facilitate sterol traffic between ER and lipid particles. The function of the other Sfh proteins is largely unsolved. Based on a detailed analysis of $\sec 14-1$ suppression by SFH2, it was suggested that $\mathrm{Sfh} 2 \mathrm{p}$ is the closest functional homolog of Sec14p [11]. Interestingly, SFH2 is the only hypoxic gene within the Sec $14 p$ family $[10,12]$. Here, we report on the possible role of $S F H 2$ in lipid and fatty acid metabolism in response to haem depletion and oleic acid starvation.

\section{EXPERIMENTAL}

\section{Yeast strains, media, genetic techniques and culture conditions}

The yeast strains used throughout this study are listed in Table 1. Strains were grown in standard minimal media (YNB) supplemented with the appropriate aminoacids and/or bases for plasmid selection. The media used for anaerobic growth were supplemented with $1 \%(\mathrm{v} / \mathrm{v})$ Tween 80 (Polyethylene Sorbitan Mono-Oleate as a source of oleic acid), $80 \mu \mathrm{g} \cdot \mathrm{ml}^{-1}$ ergosterol and 1\% (v/v) Tergitol NP-40/ethanol (1:1). In contrast to what is routinely used in the lab [1], the hem1 $\Delta$ strains was supplemented with $\delta$-aminolevulinic acid $\left(120 \mu \mathrm{g} \cdot \mathrm{ml}^{-1}\right)$, which achieved aerobic growth conditions until glucose depletion (ie stationary phase).In addition, unless otherwise stated, haem-induced lipid starved conditions were obtained by inoculating $2.10^{5}$ cells. $\mathrm{ml}^{-1}$ of stationary phase cells in YPD medium. In these conditions, expression of hypoxic genes was routinely observed 6 hours after the shift. Alternatively, YPD medium was supplemented with $80 \mu \mathrm{g} \mathrm{ml}^{-1}$ ergosterol and/or 1\% Tween $80(\mathrm{v} / \mathrm{v})$, used as the source of unsaturated fatty acids.

Plasmid pJU1 contains the SFH2 ORF and 220bp of upstream sequence inserted into Yep352 [13]. The insert was isolated as a SacI fragment from pMR270 one of the original plasmids suppressing sec14-1 phenotype [12]) into Yep352 ; we have confirmed that this plasmid allows full suppression of sec14-1 mutation and complements SFH2 deletion. Plasmid pMR306 encoding the 
fusion protein MBP-SFH2 was constructed as follows. First pMR265 [12] was used to amplify the coding sequence of $\mathrm{SFH} 2$ using the two primers CSRBGL2

(5'AAGATCTATTATGTCCTTTGATAGA3') and CSR3SAL

(5'AGTCGACTCAAACTTTTAGAGAACC3'). The two oligonucleotides introduce a BglII and a SalI restriction site immediately upstream and downstream of $S F H 2$ coding sequence. The PCR product was cloned into pGEMT-easy (Promega) to generate pMR303. The $1.3 \mathrm{~kb}$ BglII-SalI fragment from pMR303 was inserted into $\mathrm{pMAL}^{\mathrm{TM}}$-c2 (New England Biolabs Inc.) digested with SalI and BamHI to generate pMR306. Plasmid pCF8 contains FAS1 ORF under the control of the tet $_{7}$ promoter; it was constructed as follows: FASI ORF was amplified by PCR from the plasmid pJS229 (kindly provided by Pr Schüller) using the two primers 5FAS1

(5'CGCTCATTATGGACGCTTAC) and 3FAS1 (5'TTAGGATTGTTCATACTTTTC) and cloned into pGEMT-easy (Promega) to generate pGF4-S. It was digested by NotI and inserted into pCM185 to generate pCF8. This plasmid was used to transform a heterozygous FAS1/fas 14 strain from which a haploid fas $1 \Delta$ was isolated. Growth of this strain in the absence of doxycycline was indistinguishable from the wild type strain, but was totally inhibited with doxycycline concentrations above $1 \mu \mathrm{g} \cdot \mathrm{ml}^{-1}$ in the medium. This confirmed the functionality of the $\mathrm{tet}_{7}$ promoter to control accurately FAS1. Plasmid pCG4GFP is an integrative vector based on Yip351 which express GFP-Sfh2p under the control of the SFH2 promoter. It was constructed as follows: SFH2 ORF was isolated as a NotI fragment and cloned into pNG188 to generate pMR314: this plasmid contains an in frame fusion between GFP and SFH2 with a c-myc epitope in between that can be isolated as a SpeI restriction fragment. A 600 bp fragment containing SFH2 promoter was amplified by PCR and inserted into Yip351 to generate Yip-PROCSR. The terminator of PMA1 was inserted as a BamH1-Not1 fragment from plasmid pNEV-N [14]; this plasmid was named YpA. Finally, the SpeI fragment from pMR314 was inserted into YpA digested with SpeI to generate pCG4GFP. This plasmid was used to transform MRY73-6a to generate YPCG4-G9d. It was introduced into TDY36-1d by standard genetic methods.

\section{Lipid analysis and labeling}

Phospholipids and neutral lipids were analyzed using the standard procedure in use in our lab [1]. Fatty acid methyl esters obtained from bulk lipids, or each lipid class separated by TLC as described [1], were analysed by GC using a $25 \mathrm{~m}$ x $0.32 \mathrm{~mm}$ AT-1 capillary column (Alltech) with methylheptadecanoic acid as a standard. Fatty acid were quantified and used to calculate the amount of lipid in each class. The lipid content was expressed as nmol of fatty acid per $10^{9}$ (broken) cells. 
For labeling experiments with palmitic acid, yeast cells were grown in liquid medium supplemented with $\delta$-aminolevulinic acid $\left(80 \mu \mathrm{g} \cdot \mathrm{ml}^{-1}\right)$ to a cell density of $4 \times 10^{7}$ cells. $\mathrm{ml}^{-1}$ after which they were incubated with $1.5 \mu \mathrm{Ci} \cdot \mathrm{ml}^{-1}$ of $\left[9,10^{3} \mathrm{H}\right]$ palmitic acid (1.11 TBq.mmol ${ }^{-1}$, ARC Radiochemicals). After 16 hours, cells were harvested, washed with fresh non radioactive YPD medium, and chased for the indicated time $(0,4,6,8,16$ hours). At the end of the experiments, cells were recovered and mixed with $2 \times 10^{9}$ non radioactive cells. Lipids were extracted as described above. During the course of the chase experiment, there was no significant loss of radioactivity per volume of the culture within the lipid pool. The tritiated spots of individual lipid species were scraped off from the silica gel plates, transferred into scintillation counting vials and the ${ }^{3} \mathrm{H}$ content was determined by liquid scintillation counting.

\section{Enzyme activities and MBP-Sfh2 purification}

$\beta$-galactosidase assays were performed as described previously [1] on hem1 $1 \Delta$ cells containing a plasmid bearing the $1 \mathrm{~kb}$ DNA sequence immediately upstream of the DAN1 open reading frame cloned 5' to the reporter gene lacZ (plasmid pMR278 [6]), grown in the appropriate medium, or with plasmid pBF16 (ARS CEN TRP1 vector) [15] which contains FAS2 promoter and part of the coding sequence fused in frame to $l a c Z$ coding sequence. Expression of the reporter gene is given as nmol of $O$-nitrophenol (measured at $420 \mathrm{~nm}$ ) $\cdot \mathrm{min}^{-1} \cdot \mathrm{mg}^{-1}$ of protein, was calculated as follows: $\mathrm{A}=$ $(A 420 \times 100 \times 1700) /(4.5 \times V \times c \times t)$, where $A 420$ is the optical density, $V$ is the volume of the protein extract $(\mathrm{ml}), c$ is the protein concentration $\left(\mathrm{mg} \cdot \mathrm{ml}^{-1}\right)$ and $t$ is the incubation time at $30^{\circ} \mathrm{C}(\mathrm{min})$. FAS activity was determined according to Lynen [16] by assaying $\beta$-ketoacyl reductase-dependent oxidation of NADPH at $334 \mathrm{~nm}$ in the presence of acetyl-CoA and malonyl-CoA. Activity is expressed as mU.mg ${ }^{-1}$ protein (nmol NADPH oxidised per minute and per mg of protein). Cells were broken with glass beads and cleared by a 10 minutes centrifugation at $5000 \mathrm{~g}$. Depending upon the strain used, protein amounts varied between $70 \mu \mathrm{g}$ to $130 \mu \mathrm{g}$ to ensure an enzyme activity below 10 milliunits which guaranteed linearity between activity and enzyme amount [16]. MBP-Sfh2p was purified essentially as recommended by the manufacturer instruction (New England Bioloabs) with minor modifications as described elsewhere [6].

\section{Immunofluorescence Microscopy}

Liquid cultures were grown overnight in complete medium containing $\delta$-aminolevulinic acid. Cells were harvested and washed twice with distilled water and inoculated in complete media with or without $\delta$-aminolevulinic acid and cultivated for 6 hours. Cells were resuspended in PBS with $0.1 \%$ (w/v) Bovine serum albumin (SIGMA, Fraction V). Staining of lipid particles with Nile Red 
(Molecular Probes) was performed by incubating cells with Nile Red $\left(50 \mu \mathrm{g} \cdot \mathrm{ml}^{-1}\right)$ for 5 minutes, and washed in PBS 0.1\% BSA. Indirect immunofluorescence was performed from TDY33-3a using the protocol described in the TRIPLES database [17] except that mounted solution contained Vectashield 10\% (v/v) (Vector Lab Inc.) and that the antibodies that were used are : HA.11 polyclonal antibody (COVANCE) anti HA primary antibody (1:100) and Alexa Fluor ${ }^{\circledR} 488$ goat anti-rabbit $\mathrm{IgG}(\mathrm{H}+\mathrm{L})$ (Invitrogen) (1:400). Confocal microscopy was performed with the use of a Confocal Olympus FV 1000 spectral mode. Excitation wavelength for GFP and Alexa Fluor ${ }^{\circledR} 488$ were set to $488 \mathrm{~nm}$. Excitation wavelength for Nile Red was set to $543 \mathrm{~nm}$. Image acquisition and conversion were done separately for red and green channel and processed with Olympus Fluoview vers1.4. 


\section{RESULTS}

\section{Lipid composition of hem1 $1 \Delta$ fh $2 \Delta$ mutant in haem depleted medium}

To determine whether $\mathrm{SFH} 2$ is involved in lipid metabolism under haem depleted conditions a hem1 1 sfh2A mutant and the control strain (hem1A) were cultivated in complete medium supplemented with $\delta$-aminolevulinic acid to stationary phase and inoculated into fresh medium in the absence of $\delta$-aminolevulinic acid, ergosterol and oleate (i.e. haem-induced lipid starvation). Under these conditions, expression of hypoxic genes was detected 6 hours after the shift (see below) and growth ceased 16 hours after the shift due to sterol and oleic acid starvation [1]. The amount of phospholipids and neutral lipids were quantified in both strains before the shift and 16 hours later. Aerobic-like cultures of mutant and control strains grown to stationary phase had very similar profiles of phospholipids (Figure 1A). In contrast, noticeable differences in phospholipid composition were observed following oleic acid starvation and haem depletion: whereas a 53\% decrease of PtdCho was observed in the control strain, there was a 31\% increase in the mutant strain. We also noted a slight decrease of PtdIns abundance 16 hours after the shift in the mutant strain as compared to the control strain. The amount of $\mathrm{PtdOH}$, a proposed key component of phospholipid metabolism [18], was also affected since haem depletion resulted in a 2 fold increase of PtdOH amount in the mutant strain; in the control strain, it remained more or less unchanged. Finally, the amount of PtdEtn and PtdSer were similar between the two strains.

Next, we examined neutral lipid composition in the mutant strain following haem-induced lipid depletion. As reported in Figure 1B, triacylglycerol (TAG) and sterylester (SE) accumulated to the same extent in control and mutant strains cultivated to stationary phase in the presence of $\delta$ aminolevulinic acid. We observed that SE was efficiently degraded in both strains following the shift to haem induced lipid starvation. Residual amounts of SE were still detected 16 hours after the shift. It corresponded to the amount of SE detected in normal aerobic growth during exponential phase. Surprisingly, whereas $90 \%$ of the pool of TAG was depleted 16 hours following the shift in the hem $1 \Delta$ strain, $40 \%$ of the TAG accumulated in aerobiosis was still observed in the hem $1 \Delta$ sfh $2 \Delta$ (Figure 1B). We also noted a $61 \%$ increase in the amount of diacylglycerol (DAG) in the mutant strain whereas there was a 2.3 fold decrease of DAG in the control strain. 


\section{Sfh $2 \Delta$ produces more saturated fatty acids upon haem-induced lipid starvation}

We have already reported that SFA preferentially accumulate within specific phospholipid species in response to haem-induced fatty acid starvation [1]. Thus, we looked more closely at the ratio of SFA within phospholipids and neutral lipids in response to haem depletion. As reported in Table 2, the increase of SFA amount did not affect all the lipid species to the same extent. We have already shown (see Figure 1A) that haem depletion does not affect the total amount of PtdSer and PtdEtn; in Table 2, it is reported that the ratio of SFA in PtdEtn was not modified either. We noted a slight decrease of SFA ratio in PtdSer in the hem1 1 sfh $2 \Delta$ strain 16 hours after the shift as compared with the control hem1 1 . The fatty acid composition of PtdCho was not modified 16 hours after the shift between mutant and control (Table 2). However, bearing in mind that the amount of PtdCho had increased of $53 \%$ in the mutant and decreased to $35 \%$ in the control (see Figure 1A), it suggests that a substantial amount of SFA was contained in this phospholipid. In addition to a 2 fold increase in the amount of PtdOH (Figure 1A), the amount of SFA represented $72.4 \%$ of this lipid in the mutant and $46.3 \%$ in the control strain. Finally, although the amount of PtdIns had decreased in the mutant but increased in the control strain following the shift to haem depleted medium (Figure 1A), the proportion of SFA was increased to $71.6 \%$ in the mutant and to $60.0 \%$ in the control. Thus, similar amounts of SFA were contained in this phospholipid species. SFA were also preferentially stored in TAG in the mutant after the shift (they constitute $64.5 \%$ of total TAG in mutant 16 hours after the shift but only $29.2 \%$ in the control). The amount of DAG had increased of $61 \%$ in the mutant respective to the control (Figure 1A) but the proportion of SFA in this species was not dramatically altered $(84.0 \%$ in mutant and $81.6 \%$ in control). Finally, free SFA represented $41.4 \%$ of total free fatty acid in the mutant and $34.4 \%$ in the control.

To confirm that the heml $1 \Delta f h 2 \Delta$ mutant accumulates more fatty acids than the hem $1 \Delta$ control strain, the amount of fatty acids was compared between mutant and control, in response to haem-induced fatty acid depletion. As shown Figure 2A, and as already described, the amount of fatty acid in the cells rapidly decreased following inoculation in complete medium [1], because the cells preferentially reallocate their endogenous pool of fatty acid accumulated in stationary phase within lipid particles : indeed, 4 hours after inoculation from stationary phase, 2.5 generations had been accomplished, and the amount of fatty acids in the cells was 4 times lower suggesting that there is only a modest fatty acid biosynthesis following inoculation into fresh medium (see below).

Soon after the onset of hypoxic gene expression ( 6 hours), but before growth arrest of the mutant (see below, Figure 3), total fatty acids represented roughly $37.7 \%$ of the initial amount in control and $35.8 \%$ in mutant, whereas 2 hours later (i.e. 8 hours after the shift), when the mutant 
had ceased growth, it was maintained at a similar level in the control strain, but it had increased to $53.4 \%$ in the mutant strain. It is interesting to observe that in both strains, the amount of unsaturated fatty acid per $10^{9}$ cells was very similar and roughly constant during the experiment (Figure 2C). Because until 8 hours after the shift, growth of both strains was not arrested, it follows that both strains were still capable to convert newly synthesized fatty acids into unsaturated fatty acids. This is most likely the primary consequence of hypoxic induction of $O L E 1$ thereby allowing cells to desaturate fatty acid even though oxygen is limiting [19]. As shown in Figure $2 \mathrm{~B}$ and $2 \mathrm{C}$, the increase in total fatty acid content of the mutant described above can be solely accounted for by the steady increase of SFA amount.

\section{SFH2 is necessary for growth in haem depleted conditions}

To address the consequences of the lipid pattern modifications in the mutant, we compared the growth curves of the mutant strain and the wild-type in response to haem deprivation. Control hem $1 \Delta$ and mutant hem1 $s f h 2 \Delta$ were cultivated to stationary phase in the presence of $\delta$ aminolevulinate. Growth rate was monitored after a shift to fresh YPD medium without supplementation. As shown in Figure 3 and as already reported [1], haem depletion in YPD medium results in growth arrest of the control strain after about five generations (i. e. 16 hours). Hypoxic gene expression, monitored by using the lacZ reporter gene fused to the promoter of the hypoxic gene $D A N 1$, was detected 6 hours after the shift (see arrow on Figure 3) [1]. This is probably the time period necessary to deplete the cells of their endogenous pool of $\delta$-aminolevulinic acid. We observed that within 5 hours after the shift no effect of SFH2 deletion could be observed. Longer incubation in the absence of lipid supplementation (oleic acid and ergosterol) results in growth arrest of the mutant: 8 hours after the shift, cell growth was totally arrested (Figure 3). In contrast, the control strain was still able to sustain growth until 15 hours after the shift. In addition, the aberrant phenotype of the hem $1 \Delta s f h 2 \Delta$ null mutant is relieved by supplementation of the medium with oleic acid (Figure 3 ) but not by ergosterol (not shown). Thus, we conclude that oleate starvation, induced by haem depletion, results in rapid growth arrest in the absence of a functional copy of the $\mathrm{SFH} 2$ gene.

\section{Neutral lipid accumulation is not responsible of $s f h 2 \Delta$ phenotype}

Our results indicate that $\mathrm{SFH} 2$ deletion has two major consequences on lipid metabolism in response to oleic acid starvation (i) a global increase in the SFA content and (ii) an alteration of the lipid composition including an accumulation of TAG, DAG, PtdOH and PtdCho. The accumulation of TAG in lipid particles in response to $S F H 2$ deletion could be interpreted in at least two ways. It 
could simply result from a partial failure to hydrolyze neutral lipids from lipid particles.

Alternatively, neutral lipids might be efficiently degraded and used as acyl donors for phospholipid synthesis; SFH2 deletion would secondarily provoke improper storage into neutral lipids. We thus questioned the significance of TAG accumulation following haem depletion in the null SFH2 mutant.

Lipids from control hem $1 \Delta$ and hem $1 \Delta s f h 2 \Delta$ mutant growing in the presence of $\delta$ aminolevulinic acid were metabolically labeled by extensive incorporation of tritiated palmitic acid until stationary phase. Cells were then washed and inoculated in fresh YPD medium lacking $\delta$ aminolevulinic acid and oleic acid. Radioactivity within neutral lipid species separated by TLC was quantified at various time points after the shift. As shown in Figure $4 \mathrm{~A}$ and $4 \mathrm{~B}, 35.3 \pm 2.7 \%$ and $35.9 \pm 0.9 \%$ of the radioactivity incorporated into lipids before the shift was recovered in TAG in the control strain and the mutant strain respectively. SE represented $6.0 \pm 0.9 \%$ and $8.2 \pm 1.3 \%$ of total radioactivity in control and mutant respectively. We observed that 8 hours after the shift both types of molecules were effectively hydrolyzed. In addition, we could observe a concomitant increase in the radioactivity associated to phospholipids, demonstrating that acyl chains from neutral lipids had been re allocated into newly synthesized phospholipids (not shown). However, with longer chase experiments an increase in the amount of radioactivity associated to TAG was observed in the mutant strain 16 hours after the shift. In the mutant, radioactivity incorporation into TAG constituted $28.3 \pm 1.8 \%$ of total radioactivity 16 hours after the shift; in the control strain, it represented only $13.5 \pm 2.4 \%$. This led us to conclude that deletion of SFH2 leads to an improper synthesis of TAG in response to haem starvation.

To evaluate whether TAG accumulation is responsible for the premature growth arrest, the phenotype of hem1 1 sfh $2 \Delta$ inactivation was investigated in a strain background inactivated for the genes encoding TAG and SE synthases [20]. We observed that a hem1 dgal lrol arel are2 grew approximately $1 / 3$ of the optical density reached by the hem $1 \Delta$ strain (Figure 4C). This is not totally surprising since it lacked most of the fatty acids contained in the TAG and SE of the hem1 $\triangle$ strain. Nevertheless, we observed that the growth defect resulting from SFH2 deletion in haem and oleic acid depleted medium was still observed in this genetic background (Figure 4C).

Moreover, deletion of the TAG and SE synthases encoding genes (i.e. the quadruple mutant dgal lrolare1 are2), resulted in an enhanced production of some lipid species that already accumulate in the hem $1 \Delta$ sfh $2 \Delta$ mutant (i.e. PtdOH, PtdCho and DAG accumulation) without any noticeable effect on the lack of growth of this strain in haem depleted conditions (not shown). This suggests that TAG, PtdCho, DAG and PtdOH accumulation in the sfh $2 \Delta$ strain is not primarily responsible for the lack of residual growth upon haem deprivation. 


\section{The $s f h 2 \Delta$ mutant is hypersensitive to saturated fatty acids}

The results presented so far suggest that the substantial accumulation of SFA in the mutant in response to oleic acid starvation under haem depleted growth could be responsible for the hem $1 \Delta$ $s f h 2 \Delta$ phenotype. Thus, one could predict that the mutant strain would be more sensitive to exogenous SFA than the control strain under haem depleted conditions. To address this possibility, hem1 $\Delta$ sfh $2 \Delta$ and hem1 $\Delta$ strains were cultivated to stationary phase in the presence of $\delta$ aminolevulinic acid and shifted to complete medium containing a limited amount of Tween 80 as a source of oleic acid and variable amounts of Tween 40 as a source of palmitic acid. The optical density reached by the cultures was measured 16 hours later. An example, representative of three independent experiments, is presented in Figure 5. A limited amount of Tween $80(1 \%$ v $/ \mathrm{v})$ could significantly increase the growth of control and mutant strains. As expected the hem $1 \Delta s f h 2 \Delta$ was much more sensitive to addition of saturated fatty acids than the hem $1 \Delta$ strain: indeed, the presence of Tween $40(0.5 \%$ o00 $\mathrm{v} / \mathrm{v})$ in the medium had no detectable effect on the hem1 1 strain while it reduced growth of the hem $1 \Delta s f h 2 \Delta$ strain to $35 \%$ of what is observed in the absence of Tween 40 . The amount of Tween 40 necessary to inhibit growth despite the supplementation with $1 \% 000(\mathrm{v} / \mathrm{v})$ oleate was roughly 3 times less in the hem $1 \Delta$ sfh $2 \Delta$ than with the hem $1 \Delta$ strain $(2 \% 000$ and $6 \%$ respectively). Therefore, the growth defect of the mutant is exacerbated by SFA supplementation suggesting a deleterious effect. This confirms that growth inhibition of the hem $1 \Delta s f h 2 \Delta$ mutant results from endogenous accumulation of SFA.

\section{Enhanced activity of fatty acid synthase in $s f h 2 \Delta$ in vivo}

Thus, we tried to understand the basis of SFA accumulation in the mutant in response to oleic acid deprivation. In yeast, SFA are synthesized by a heteromultimeric $\alpha_{6} \beta_{6}$ complex encoded by the two genes $F A S 1$ and $F A S 2$ for the $\beta$ and $\alpha$ subunits respectively [21]. Both genes are regulated similarly and appear to be down regulated by exogenous SFA in the culture medium [22]. We wondered whether oleic acid could also control FAS expression. For this purpose, we used a lac $Z$ gene reporter assay to monitor FAS2 expression in our growth conditions (plasmid pBF16 [15] kindly provided by Pr H.J. Schüller). To minimize possible interference from the genetic background, the experiments were performed in a hem $1 \Delta s f h 2 \Delta$ mutant transformed either with the control plasmid (YEp352) or with a plasmid expressing SFH2 (pJU1). As reported in Figure 6, control and mutant strains behaved very similarly : addition of oleic acid in the medium in the presence of $\delta$ aminolevulinate resulted in a 2.6 fold decrease of the reporter gene activity, a level of repression in the same range as with a SFA (palmitic acid : 2.9 fold decrease). In contrast, we observed a 1.8 fold 
induction of the reporter gene under haem and oleic acid starvation. Importantly, supplementation with oleic acid in the absence of haem reduced FAS2 expression 3.1 fold, to a level of expression below that observed in the presence of haem. Therefore, oleic acid starvation in cells lacking haem results in enhanced expression of FAS2.

This last observation was somewhat surprising since, although both strains regulate transcription of FAS2 identically, the mutant only showed an increase in the amount of SFA in response to haem depletion and oleic acid starvation. Thus, we measured the activity of fatty acid synthase in the hem $1 \Delta s f h 2 \Delta$ and in the hem $1 \Delta$ strains in response to haem depletion. As shown in Figure 7, fatty acid synthase (FAS) activity in cells grown to stationary phase in aerobiosis was similar in both strains $\left(28.8 \pm 2.9 \mathrm{mU}_{\mathrm{mg}} \mathrm{m}^{-1}\right.$ and $23.4 \pm 3.2 \mathrm{mU} . \mathrm{mg}^{-1}$ respectively $)$. In the hem $1 \Delta$, FAS activity was maintained at a low level following oleic acid starvation in haem depleted medium: 10 hours after the shift, FAS activity was $31.9 \pm 0.1 \mathrm{mU} \cdot \mathrm{mg}^{-1}$. In contrast, in the hem1 $s f h 2 \Delta$ strain, haem depletion and oleic acid starvation were associated with an increase of FAS activity $\left(57.87 \pm 4.7 \mathrm{mU} . \mathrm{mg}^{-1}\right.$ detected 6 hours after the shift) which was maintained at a high level during the rest of the experiment.

\section{Suppression of $s \boldsymbol{f h} 2 \Delta$ phenotype by controlled expression of FAS1}

It has been shown that the activity of FAS holoenzyme is primarily determined by the amount of Fas $1 \mathrm{p}$ since overexpression of FAS1 gene results in concomitant increase of FAS2 mRNA abundance and an enhanced activity of FAS [15]. Thus, it is possible to alter the amount of active FAS by controlling the transcriptional level of the sole FAS1 gene. This observation provided a convenient way to test whether increased FAS activity is responsible for the phenotype of the hem1 1 sfh2 $\Delta$ strain. Plasmid pCF8 contains an engineered FAS1 gene whose expression is controlled by the $t^{2} O_{7}-\mathrm{CYC} 1$ promoter which is negatively regulated by doxycycline [23]. It was used to transform a heterozygous FAS1/fas 14 strain from which a viable haploid fas $1 \triangle$ was isolated. In this strain, expression of the episomal $F A S 1$ gene is driven by the tet $_{7}-\mathrm{CYC} 1$ promoter and consequently FAS activity is modulated by the amount of doxycycline added to the medium as demonstrated for other genes [24] : indeed, we observed that fas $1 \Delta$ [pCF8] strain in aerobiosis is viable, and its growth is inhibited by doxycycline concentrations above $1 \mu \mathrm{g} \cdot \mathrm{ml}^{-1}$ because expression of the engineered FAS1 gene is totally repressed and FAS activity is undetectable (not shown).

Then, we constructed hem $1 \Delta$ fas $1 \Delta$ and heml $1 \Delta$ fas $1 \Delta$ sfh $2 \Delta$ strains containing plasmid pCF 8 ; both strains were cultivated in the presence of $\delta$-aminolevulinic acid to stationary phase and further inoculated in complete medium with variable amounts of doxycycline in the medium in 
order to control FAS activity. The optical density reached by the cultures was measured 40 hours after the shift. As shown in Figure 8A, in the absence of doxycycline in the medium, or with concentration of doxycycline above $0.4 \mu \mathrm{g} \cdot \mathrm{ml}^{-1}$, the mean optical densities reached by the strains $(0.186 \pm 0.046$ and $0.184 \pm 0.004$ for hem $1 \Delta$ fas $1 \Delta$ [pCF8] and hem $1 \Delta$ fas $1 \Delta$ sfh2 4 [pCF8] strains respectively) suggest that residual growth was limited, if not totally impaired. Indeed, the hem $1 \Delta$ $s f h 2 \Delta$ mutant attained similar values under these growth conditions (not shown). In contrast, with $0.15 \mu \mathrm{g} \cdot \mathrm{ml}^{-1}$ of doxycycline, a significant residual growth was attained with the hem1 1 fas $1 \Delta$ : indeed, the optical density achieved for this strain was $0.474 \pm 0.02$ which is only slightly lower than for a hem1 $\Delta$ strain $\left(0.508 \pm 0.09\right.$ obtained with a similar inoculum $\left(2 \times 10^{5} \mathrm{cells} . \mathrm{ml}^{-1}\right)$, not shown). Interestingly, a higher concentration of doxycycline was needed for the hem $1 \Delta$ fas $1 \Delta$ sfh $2 \Delta$ strain to achieve a significant residual growth $\left(\mathrm{OD}_{600 \mathrm{~mm}}: 0.391 \pm 0.05\right.$ with doxycycline concentration of $0.30 \mu \mathrm{g} \cdot \mathrm{ml}^{-1}$ ). FAS activity was also measured in both strains after the shift (Figure 8B). We observed that, for any concentration of doxycycline, FAS activity detected in the hem $1 \Delta$ fas $1 \Delta$ [pCF8] strain was always lower than in the hem1 1 fas $1 \Delta \mathrm{sfh} 2 \Delta$ [pCF8] strain. This confirms the up regulation of FAS activity in the $s f h 2$ null mutant. To get better insight in those activities, it is useful to compare with values obtained in hem1 $1 \mathrm{sfh} 2 \Delta$ and hem1 1 strains - in which FAS activity depends on chromosomal expression of FAS genes - in response to haem depletion and oleic acid starvation (Figure 8C). FAS activity close to that obtained in the hem $1 \Delta$ strain were observed with doxycycline concentrations compatible with significant residual growth (i.e. $0.15 \mu \mathrm{g} \cdot \mathrm{ml}^{-1}$ and $0.30 \mu \mathrm{g} \cdot \mathrm{ml}^{-1}$ for hem1 $\Delta$ fas $1 \Delta$ [pCF8] and hem $1 \Delta$ fas $1 \Delta$ sfh $2 \Delta$ [pCF8] respectively). In contrast, with lower amounts of doxycycline in the medium, values of FAS activities were closer (or even higher) to values obtained in the hem $1 \Delta s f h 2 \Delta:$ in these growth conditions, no residual growth was observed in the corresponding strains as a consequence of overexpression of episomal FAS1 in the hem1 fas1 $[\mathrm{pCF} 8]$ strain. Thus, these experiments demonstrate that down regulating FAS1 transcription is sufficient to suppress the phenotype of hem1 $14 \mathrm{fh} 2 \Delta$. In addition, the high amount of doxycycline necessary for the hem1 4 fas $1 \Delta s f h 2 \Delta$ [pCF8] to achieve residual growth is consistent with an already higher FAS activity in the triple mutant strain than in the hem1 $\Delta$ fas $1 \Delta$ and confirms that residual growth necessitates a tight regulation of FAS activity by Sfh2p.

\section{Sfh2p inhibits fatty acid synthase activity}

The elevated FAS activity in vivo in the absence of oleic acid and SFH2 led us to investigate the possibility that Sfh2p inhibits the activity of FAS. For this purpose, we engineered an N-terminal tagged version of Sfh2p, namely, MBP-Sfh2p. We measured the effect of adding purified recombinant $\mathrm{Sfh} 2 \mathrm{p}$ on FAS activity in vitro. In this assay, cellular extracts were prepared from the 
hem1 1 sfh $2 \Delta$ mutant incubated for 6 hours in a $\delta$-aminolevulinic and oleic acid free complete medium (i.e. high level of FAS activity). Various amount of recombinant MBP-Sfh2p purified from E. coli were incubated in the assay medium 1 minute before initiating the reaction. As shown Figure 9, recombinant $\mathrm{Sfh} 2 \mathrm{p}$ could substantially inhibit FAS activity (up to 50\% inhibition). In contrast, addition of $150 \mathrm{ng}$ of purified MBP in the assay did not affect FAS activity significantly (not shown). We have already mentioned that in aerobiosis, deletion of $S F H 2$ resulted in a $30 \%$ increase of FAS activity. Addition of recombinant Sfh2p to cell extracts of the $s f h 2 \Delta$ mutant grown in aerobiosis restored the activity to a wild type level, suggesting that under aerobic growth, Sfh $2 p$ could also be used to regulate FAS activity (not shown).

\section{Sfh2p is localized in lipid particles}

The cellular localization of Sfh2p is still a matter of debate. It was reported to localize, at least partially, to endosomes [10]. In contrast, Schnabl et al. reported that Sfh2p localized mainly in the cytosol and microsomes [11]. In order to address the subcellular localization of Sfh2p, we used an integrated version of SFH2 which expresses a GFP-tagged Sfh2p. The fluorescent tag was inserted at the N-terminus of Sfh2p and was functional as judged by the ability of the recombinant GFP$\mathrm{Sfh} 2 \mathrm{p}$ to rescue the phenotype of hem $1 \Delta s f h 2 \Delta$ mutation (not shown). The recombinant strain was grown to stationary phase in the presence of $\delta$-aminolevulinic acid. GFP-Sfh $2 p$ localized to several punctuated structures scattered in the cytoplasm (see Figure 10 left panel). Interestingly, this pattern was similar to the one observed for the fluorescent dye Nile Red, which is specific for neutral lipids, and is used as a marker of lipid particles. Computer merge of these images confirmed the localization of GFP-Sfh2p with Nile Red structures (not shown). We estimated that more than 90\% of the GFP-Sfh2p positive structures were also positive with Nile Red. Following inoculation in $\delta$ aminolevulinic depleted medium and in the absence of oleic acid, the pattern of GFP-Sfh2p remained very similar, suggesting that the protein remained associated with lipid droplets (not shown). In contrast, in the presence of $\delta$-aminolevulinic acid in the medium, the signal promptly faded and 6 hours after the shift, the protein was not detectable anymore (not shown), as expected for the product of a hypoxic gene whose level of expression in exponential phase in aerobiosis is low [12].

We also tried to localize Fas $1 p$ in the cell. For this purpose, we used a strain expressing a functional Fas 1p-3xHA fusion protein that was kindly provided by Dr Snyder (TRIPLE database) from which a hem $1 \Delta$ derivative was obtained. Indirect immunofluorescence using anti-HA monoclonal antibodies was performed from the strain grown in a medium supplemented with $\delta$ aminolevulinic acid. In exponential growth, the signal was diffuse in the cell (not shown), 
suggesting a cytosolic distribution as already described for this protein. In contrast, sample from stationary phase cells revealed a very different localization of Fas1p-3xHA (Figure 10B, left panel): the fluorescent signal was localized in discrete punctuate structures, which again correspond to lipid particles as judged by the co-localization with Nile Red (Figure 10B, middle panel). Inoculation of this strain in complete medium lacking $\delta$-aminolevulinic acid and oleic acid did not modify the localization of Fas1p-3xHA (Figure 10C) which was still associated with lipid particles 6 hours after the shift. In contrast, in the presence of $\delta$-aminolevulinic acid in the medium, the fluorescent signal was not associated with lipid droplets (Figure 10D), suggesting a cytosolic distribution of this protein. Nevertheless, under haem depletion and oleic acid starvation, both Sfh2p and Fas $1 p$ appeared to be co-localized in lipid particles. Interestingly, this localization was not modified in a $s f h 2 \Delta$ null mutant (unpublished results). All together, genetic data and localization data support the notion that Sfh2p negatively regulates FAS.

\section{DISCUSSION}

Saccharomyces cerevisiae is only able to synthesize monounsaturated fatty acids containing a $\Delta-9$ double bond, primarily palmitoleic acid (C16:1) and oleic acid (C18:1) which represent respectively around $45 \%$ and $25 \%$ of total fatty acids in aerobiosis. The $\Delta-9$ desaturase responsible for the synthesis of these unsaturated fatty acids (UFA) is encoded by a single essential gene, OLE1. In aerobiosis, transcriptional regulation of $O L E 1$, and modulation of $O L E 1$ mRNA stability maintain an adequate balance of UFA versus SFA [25]. Since Ole1p is strictly dependent on haem availability, hence on oxygen status, life under hypoxic or anoxic conditions has important consequences on this ratio. Accordingly, when the oxygen status is low in the cell, induction of $F A S$ genes and of $O L E 1$ facilitates desaturation of both existing and newly synthesized fatty acids as long as oxygen is still available [26]. However in strict anaerobiosis or in haem depleted conditions, i.e. when Ole1p is not functional $F A S$ genes transcription is still observed if fatty acids are not provided in the medium, and import of exogenous UFA from the medium becomes strictly necessary for optimal growth.

In this work we report a body of evidence which indicates that $\mathrm{Sfh} 2 \mathrm{p}$ modulates FAS activity in response to haem depletion and oleate starvation and that, under this condition, this regulation is essential to prevent SFA accumulation that eventually leads to premature growth arrest. Consistently, $s f h 2 \Delta$ displays enhanced sensitivity to SFA in the medium whereas UFA supplementation restores wild type phenotype. In agreement with a detrimental effect of $S F H 2$ 
deletion on FAS activity, we observed an increase of FAS activity in the mutant in response to haem depletion and UFA starvation. This is confirmed by the observation that $s f h 2 \Delta$ can be rescued by downregulation of $F A S 1$ gene expression while enhanced expression of FAS1 gene generates a phenotype which is similar to $s f h 2 \Delta$ (see Figure 8). However, the mechanism of FAS inhibition by $\mathrm{Sfh} 2 \mathrm{p}$ is still unclear. We would tend to favour a model by which $\mathrm{Sfh} 2 \mathrm{p}$ exerts a direct effect on FAS because ( $i$ ) in vitro, addition of purified Sfh2p inhibits FAS activity (ii) both proteins colocalize within lipid particles and, (iii) we have observed in vitro that Sfh2p can bind Fas $1 \mathrm{p}$ (unpublished results). However we have not yet been able to confirm this interaction in vivo.

We also observed that residual growth of $s f h 2 \Delta$ mutant is impaired in genuine anaerobiosis in the absence of oleic acid supplementation (unpublished results) suggesting that Sfh2p accomplish the same function under genuine anaerobiosis as in response to haem depletion. A possible way to understand the physiological relevance of $\mathrm{Sfh} 2 \mathrm{p}$ function in response to oxygen depletion is to examine the phenotype resulting from altered expression of FAS (Figure 8). We observed that neither high nor low FAS activity can restore residual growth in response to haem depletion and UFA starvation. This is very surprising since there is a sharp transcriptional induction of $F A S$ genes in the absence of oleic acid and haem (see Figure 6). These apparent conflicting results can be reconciled bearing in mind that when oxygen becomes limited, there is transcriptional derepression of hypoxic genes, including $O L E 1$, and also FAS1 and FAS2 if fatty acid are not present in the medium. As long as Olelp is active (i.e. oxygen is still present in the cell), the adequate balance between saturated and unsaturated fatty acids is still guaranteed in the cell. However, as soon as haem synthesis ceases consequently to oxygen depletion, fatty acid desaturation by Ole1p is precluded whereas FAS is still abundant and active in the absence of fatty acid supplementation. We propose that the function of Sfh2 $\mathrm{p}$ is to inhibit FAS under this condition and that this fine tuning of FAS by Sfh 2 p would allow the cell to prevent the deleterious accumulation of saturated fatty acid. As a Sec14p related protein, Sfh2p contains a hydrophobic pocket that can potentially bind phospholipids. It is tempting to speculate that inhibition of FAS activity by Sfh $2 p$ might be controlled by a lipid bound form. Amongst the lipids that accumulate in a hem $1 \Delta$ sfh $2 \Delta$ in response to haem depletion and UFA starvation, PtdOH fulfils several criteria to be a candidate to accomplish this function. Indeed, not only does this lipid accumulate in response to haem and UFA depletion, but it is also highly enriched in SFA (see Table2). Moreover it is produced in lipid particles [27] i.e. a compartment where Sfh2p and Fas $1 p$ are mainly localised in response to haem depletion. Further work is needed in order to test this hypothesis. 
SFH2 encodes a gene which was originally identified in a screen for multicopy suppressors of the double mutant chs 5 spa2 defective in chitin synthesis and cellular morphology (Santos and Snyder, 2000). It was also observed that it can physically interact with a thiol peroxidase type II isoform (cTPx II ) in vitro [28] although the functional significance of this interaction is unclear with the function of Sfh2 $\mathrm{p}$ as described in this report. Most interestingly, in addition to the sequence homology between Sfh2p and Sec14p, a protein involved in the regulation of phospholipid metabolism, it was observed that $S F H 2$ was also a multicopy suppressor of SEC14 loss of function $[10,12]$. A decrease in FAS activity might be relevant to understand the mechanism of suppression of sec14 4 by SFH2 overexpression. Indeed, we observed an increase in FAS activity in a sec14-1 mutant at the restrictive temperature which was restored in response to SFH2 overexpression (unpublished observations). Sec14p controls PtdCho metabolism by inhibiting Pctlp, the rate-limiting enzyme of the CDP-choline pathway for PtdCho synthesis. Recently, it was also demonstrated that Sec14p is involved in PtdCho degradation by stimulating Nte1p, a phospholipase B specific for PtdCho [29, 30]. Therefore, PtdCho level in yeast is controlled both at the level of its synthesis and at the level of its degradation. Interestingly, we observed that deletion of $\mathrm{SFH} 2$ which stimulates FAS activity results in a significant accumulation of PtdCho in haem and oleic acid depleted medium (Figure 1). This suggests that FAS activity impinges on PtdCho biosynthesis and/or degradation. This view is supported by data obtained from CHO cells demonstrating that inhibition of FAS activity by the antibiotic cerulenin impairs PtdCho biosynthesis $[31,32]$. These authors observed that this effect is mediated by an inhibition of Pct1p. Although it is tempting to speculate that in yeast, fatty acid synthesis inhibition, induced by Sfh2p, could lead to Pctlp inhibition and thus facilitate a by-pass Sec14p phenotype, this view is not fully supported by experimental data. Indeed, $S F H 2$ overexpression in the absence of SEC14 does not seem to affect PtdCho degradation [29]. However, a significantly diminished rate of choline uptake, an accumulation of cytoplasmic choline, and an altered P-Cho:Cho ratio in the cells toward a lower amount of P-Cho versus Cho were observed in response to SFH2 overexpression in a sec14A mutant. This suggests that the metabolic flux in the Kennedy pathway is diminished, but not through inhibition of Pct1p (which would then result in an increase of P-Cho at the expense of choline). One attractive possibility would be that this reduced flux is a consequence of the limited availability of the substrates and more specifically of reduced synthesis of DAG as a consequence of FAS inhibition. The observation that DAG also accumulates in the absence of $\mathrm{SFH} 2$, in haem and oleic acid depleted medium (see Figure 1), is consistent with this hypothesis. 


\section{ACKNOWLEDGEMENTS}

This work was supported by the french MENRT and CNRS. TD was supported by a grant from MENRT. We would like to gratefully acknowledge Pr H.J. Schüller (Universität Greifswald, Germany) for generously providing us plasmid pBF16 and pJS229, and members of the Snyder lab for providing yeast strain V83B2. François Doignon (Université de Bordeaux II, France) is kindly acknowledged for providing pCM185, Jean Marc Galan (Université de Paris VI et VII) for providing pNG188. Anne Cantereau is acknowledged for her technical assistance with confocal microscopy. 


\section{REFERENCES}

1 Ferreira, T., Regnacq, M., Alimardani, P., Moreau-Vauzelle, C. and Berges, T. (2004) Lipid dynamics in yeast under haem-induced unsaturated fatty acid and/or sterol depletion. Biochem. J. 378, 899-908

2 Kwast, K. E., Lai, L. C., Menda, N., James, D. T., 3rd, Aref, S. and Burke, P. V. (2002) Genomic analyses of anaerobically induced genes in Saccharomyces cerevisiae: functional roles of Rox 1 and other factors in mediating the anoxic response. J. Bacteriol. 184, 250-265

3 ter Linde, J. J., Liang, H., Davis, R. W., Steensma, H. Y., van Dijken, J. P. and Pronk, J. T. (1999) Genome-wide transcriptional analysis of aerobic and anaerobic chemostat cultures of Saccharomyces cerevisiae. J. Bacteriol. 181, 7409-7413

4 Ter Linde, J. J. and Steensma, H. Y. (2002) A microarray-assisted screen for potential Hap1 and Rox1 target genes in Saccharomyces cerevisiae. Yeast 19, 825-840

5 Zhang, L. and Hach, A. (1999) Molecular mechanism of heme signaling in yeast: the transcriptional activator Hap1 serves as the key mediator. Cell. Mol. Life Sci. 56, 415426

6 Regnacq, M., Alimardani, P., El Moudni, B. and Berges, T. (2001) SUT1p interaction with Cyc8p(Ssn6p) relieves hypoxic genes from Cyc8p-Tup $1 p$ repression in Saccharomyces cerevisiae. Mol. Microbiol. 40, 1085-1096

7 Reiner, S., Micolod, D., Zellnig, G. and Schneiter, R. (2006) A genomewide screen reveals a role of mitochondria in anaerobic uptake of sterols in yeast. Mol. Biol. Cell 17, $90-103$

8 Routt, S. M. and Bankaitis, V.A. (2004) Biological functions of phosphatidylinositol transfer proteins. Biochem. Cell. Biol. 82, 254-262

9 Skinner, H. B., McGee, T. P., McMaster, C. R., Fry, M. R., Bell, R. M. and Bankaitis, V. A. (1995) The Saccharomyces cerevisiae phosphatidylinositol-transfer protein effects a ligand-dependent inhibition of choline-phosphate cytidylyltransferase activity. Proc. Natl. Acad. Sci. U. S. A. 92, 112-116

10 Li, X., Routt, S. M., Xie, Z., Cui, X., Fang, M., Kearns, M. A., Bard, M., Kirsch, D. R. and Bankaitis, V. A. (2000) Identification of a novel family of nonclassic yeast phosphatidylinositol transfer proteins whose function modulates phospholipase D activity and Sec14p-independent cell growth. Mol. Biol. Cell 11, 1989-2005 
11 Schnabl, M., Oskolkova, O. V., Holic, R., Brezna, B., Pichler, H., Zagorsek, M., Kohlwein, S. D., Paltauf, F., Daum, G. and Griac, P. (2003) Subcellular localization of yeast Sec14 homologues and their involvement in regulation of phospholipid turnover. Eur. J. Biochem. 270, 3133-3145

12 Regnacq, M., Ferreira, T., Puard, J. and Berges, T. (2002) SUT1 suppresses sec14-1 through upregulation of CSR1 in Saccharomyces cerevisiae. FEMS Microbiol. Lett. 216, $165-170$

13 Hill, J. E., Myers, A. M., Koerner, T. J. and Tzagoloff, A. (1986) Yeast/E. coli shuttle vectors with multiple unique restriction sites. Yeast 2, 163-167

14 Sauer, N. and Stolz, J. (1994) SUC1 and SUC2: two sucrose transporters from Arabidopsis thaliana; expression and characterization in baker's yeast and identification of the histidine-tagged protein. Plant J. 6, 67-77

15 Wenz, P., Schwank, S., Hoja, U. and Schuller, H. J. (2001) A downstream regulatory element located within the coding sequence mediates autoregulated expression of the yeast fatty acid synthase gene FAS2 by the FAS1 gene product. Nucleic Acids Res. 29, 4625-4632

16 Lynen F. (1969) Yeast Fatty Acid Synthase. Methods in Enzmology XIV, 17-33

17 Burns, N., Grimwade, B., Ross-Macdonald, P. B., Choi, E. Y., Finberg, K., Roeder, G. S. and Snyder, M. (1994) Large-scale analysis of gene expression, protein localization, and gene disruption in Saccharomyces cerevisiae. Genes Dev. 8, 1087-1105

18 Loewen, C. J., Gaspar, M. L., Jesch, S. A., Delon, C., Ktistakis, N. T., Henry, S. A. and Levine, T. P. (2004) Phospholipid metabolism regulated by a transcription factor sensing phosphatidic acid. Science 304, 1644-1647

19 Zitomer, R. S., Carrico, P. and Deckert, J. (1997) Regulation of hypoxic gene expression in yeast. Kidney Int. 51, 507-513

20 Sandager, L., Gustavsson, M. H., Stahl, U., Dahlqvist, A., Wiberg, E., Banas, A., Lenman, M., Ronne, H. and Stymne, S. (2002) Storage lipid synthesis is non-essential in yeast. J. Biol. Chem. 277, 6478-6482

21 Schweizer, E. and Hofmann, J. (2004) Microbial type I fatty acid synthases (FAS): major players in a network of cellular FAS systems. Microbiol. Mol. Biol. Rev. 68, $501-517$

22 Chirala, S. S. (1992) Coordinated regulation and inositol-mediated and fatty acidmediated repression of fatty acid synthase genes in Saccharomyces cerevisiae. Proc. Natl. Acad. Sci. U. S. A. 89, 10232-10236 
23 Gari, E., Piedrafita, L., Aldea, M. and Herrero, E. (1997) A set of vectors with a tetracycline-regulatable promoter system for modulated gene expression in Saccharomyces cerevisiae. Yeast 13, 837-848

24 Cabiscol, E., Belli, G., Tamarit, J., Echave, P., Herrero, E. and Ros, J. (2002) Mitochondrial Hsp60, resistance to oxidative stress, and the labile iron pool are closely connected in Saccharomyces cerevisiae. J. Biol. Chem. 277, 44531-44538

25 Gonzalez, C. I. and Martin, C. E. (1996) Fatty acid-responsive control of mRNA stability. Unsaturated fatty acid-induced degradation of the Saccharomyces OLE1 transcript. J. Biol. Chem. 271, 25801-25809

26 Jiang, Y., Vasconcelles, M. J., Wretzel, S., Light, A., Martin, C. E. and Goldberg, M. A. (2001) MGA2 is involved in the low-oxygen response element-dependent hypoxic induction of genes in Saccharomyces cerevisiae. Mol. Cell. Biol. 21, 6161-6169

27 Athenstaedt, K. and Daum, G. (1997) Biosynthesis of phosphatidic acid in lipid particles and endoplasmic reticulum of Saccharomyces cerevisiae. J. Bacteriol. 179, $7611-7616$

28 Cha, M. K., Hong, S. K., Oh, Y. M. and Kim, I. H. (2003) The protein interaction of Saccharomyces cerevisiae cytoplasmic thiol peroxidase II with SFH2p and its in vivo function. J. Biol. Chem. 278, 34952-34958

29 Murray, J. P. and McMaster, C. R. (2005) Nte1p-mediated deacylation of phosphatidylcholine functionally interacts with Sec14p. J. Biol. Chem. 280, 85448552

30 Zaccheo, O., Dinsdale, D., Meacock, P. A. and Glynn, P. (2004) Neuropathy target esterase and its yeast homologue degrade phosphatidylcholine to glycerophosphocholine in living cells. J. Biol. Chem. 279, 24024-24033

31 Lagace, T. A., Storey, M. K. and Ridgway, N. D. (2000) Regulation of phosphatidylcholine metabolism in Chinese hamster ovary cells by the sterol regulatory element-binding protein (SREBP)/SREBP cleavage-activating protein pathway. J. Biol. Chem. 275, 14367-14374

32 Ridgway, N. D. and Lagace, T. A. (2003) Regulation of the CDP-choline pathway by sterol regulatory element binding proteins involves transcriptional and posttranscriptional mechanisms. Biochem. J. 372, 811-819

33 Winston, F., Dollard, C. and Ricupero-Hovasse, S. L. (1995) Construction of a set of convenient Saccharomyces cerevisiae strains that are isogenic to S288C. Yeast 11, 5355 
B Biochemical Journal Immediate Publication. Published on 6 Sep 2007 as manuscript BJ20071028

34 Ness, F., Achstetter, T., Duport, C., Karst, F., Spagnoli, R. and Degryse, E. (1998) Sterol uptake in Saccharomyces cerevisiae heme auxotrophic mutants is affected by ergosterol and oleate but not by palmitoleate or by sterol esterification. J. Bacteriol. 180, 1913-1919 


\section{Figure Legends}

Figure 1. Oleic acid starvation and haem depletion induce accumulation of specific lipids in the absence of functional $\mathrm{SFH} 2$.

Haem-induced lipid starved conditions, as described under Experimental procedures, were realized for 16 hours with the control strain MRY72 (hem14) and the mutant strain MRY82-2 (hem14 $s f h 24)$. (A) Fatty acids derived from phospholipids were quantified from control strain grown in aerobiosis to stationary phase in the presence of $\delta$-aminolevulinic acid or 16 hours after a shift in complete medium in the absence of $\delta$-aminolevulinic and oleic acid. (B) Same as above except that fatty acids derived from neutral lipids and free fatty acids (FFA) were quantified. For (A) and (B), data are expressed as nmol of fatty acid per $10^{9}$ cells $\pm \mathrm{SD}$ and represent the average of at least two independent experiments.

Figure 2. Increased amount of saturated fatty acids in haem depleted conditions and in the absence of oleate is observed in the $s f h 24$.

Yeast strains MRY72 (hem14) and MRY82-2 (hem1 1 sfh24) were cultivated in complete medium supplemented with $\delta$-aminolevulinic acid to stationary phase. At time zero, cells were washed and re-inoculated in complete medium lacking $\delta$-aminolevulinic and oleic acid (haem induced UFA starvation). (A) Total amount of fatty acid, (B) saturated fatty acids and (C) unsaturated fatty acids were quantified as a function of time after the shift. The data represent the average \pm SD of at least two independent experiments.

Figure 3. Oleic acid starvation and haem depletion impair growth of the hem1A sfh $2 \Delta$ mutant. Yeast strains MRY72 (hem14) (open symbols) and MRY82-2 (hem1A sfh24) (filled symbols) were grown in complete medium containing $\delta$-aminolevulinic acid to stationary phase. At time 0 , they were harvested, washed with distilled water, and $10^{6}$ cells were inoculated per ml of YPD medium with (square) or without (circle) (oleic acid). Growth was measured by optical density at $600 \mathrm{~nm}$. Vertical arrow indicates the time of induction of hypoxic gene expression using the promoter of $D A N 1$ fused to lacZ as a reporter gene [1].

Figure 4. TAG and SE are efficiently mobilized in the absence of $\mathbf{S F H} 2$.

Yeast strains MRY72 (hem14) and MRY82-2 (hem1 1 sfh2A) were cultivated in complete medium supplemented with $\delta$-aminolevulinic acid to stationary phase in the presence of $1.5 \mu \mathrm{Ci} \cdot \mathrm{ml}^{-1}$ of $\left[{ }^{3} \mathrm{H}\right]$ palmitic acid. At time zero, cells were washed and re-inoculated into non radioactive complete 
medium lacking $\delta$-aminolevulinic and oleic acid. (A) Radioactivity in TAG at time zero, 6 hours, 8 hours and 16 hours after the shift. (B) Radioactivity in SE at time zero, 6 hours, 8 hours and 16 hours after the shift. Data are expressed as percentage of total label within lipids at each time-point. The data represent the average \pm SD of at least two independent experiments. (C) Yeast strains

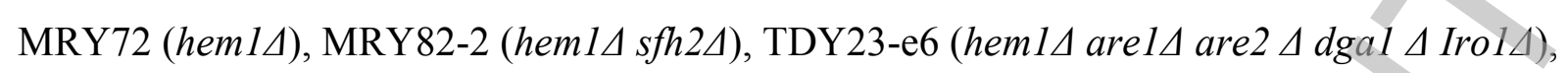

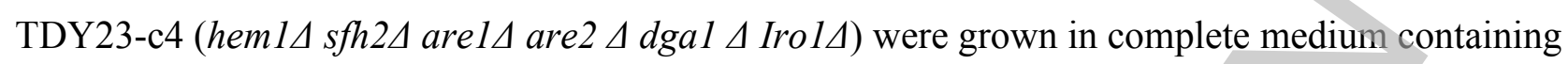
$\delta$-aminolevulinic acid to stationary phase. At time 0 , there were harvested, washed with distilled water, and $10^{6}$ cells were inoculated per $\mathrm{ml}$ of YPD medium. Growth was measured by optical density at $600 \mathrm{~nm}$. Vertical arrow indicates the time of induction of hypoxic gene expression using the promoter of $D A N 1$ fused to $l a c Z$ as a reporter gene [1].

\section{Figure 5. The mutant hem1 $s f h 2 \Delta$ is hypersensitive to SFA.}

Yeast strains MRY72 (hem14) and MRY82-2 (hem1A sfh2A) were cultivated in complete medium supplemented with $\delta$-aminolevulinic acid to stationary phase. Cells were washed and re-inoculated in complete medium supplemented with the indicated amount of Tween 40 (as a source of palmitic acid) or Tween 80 (as a source of oleic acid). The optical density of the culture was measured 16 hours after the shift.

\section{Figure 6. Oleic acid mediated control of $F A S 2$ transcription.}

Reporter plasmid pBF16 (FAS2-lacZ ARS CEN TRP1) was transformed into MRY82-2 (containing plasmid pJU1 (SFH2) or control vector (Yep352)). Transformants were inoculated in minimal medium supplemented with $\delta$-aminolevulinic acid to stationary phase. Then, they were inoculated in complete medium with supplementation as indicated for 6 hours. Cells were collected, washed and $\beta$-galactosidase activity was measured. Data are the value \pm SD of at least two experiments. Tween 80 (as a source of oleic acid) and Tween 40 (as a source of palmitic acid) were provided at a concentration of $1 \%(\mathrm{v} / \mathrm{v})$.

Figure 7. The mutant hem1 $1 \mathrm{sfh} 2 \Delta$ does not appropriately control fatty acid synthase activity in haem and oleic acid depleted medium.

Yeast strains MRY72 (hem1 4 ) and MRY82-2 (hem1 sfh24) were processed as described in Figure

3. Specific fatty acid synthase activity was measured as a function of time following the shift in $\delta$ aminolevulinate and oleic acid depleted medium. Specific activity is expressed as nmol NADPH 
oxidized per minute per $\mathrm{mg}$ of protein $\left(\mathrm{mU} \cdot \mathrm{mg}^{-1}\right)$. Values are the mean $\pm \mathrm{SD}$ of at least two experiments.

Figure 8. Controlled expression of $F A S 1$ suppresses $s f h 2 \triangle$ phenotype.

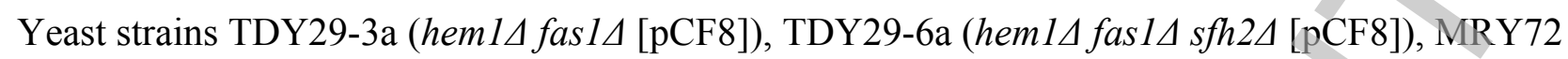
(hem14) and MRY82-2 (hem1 $\Delta$ sfh24) were grown in complete medium containing $\delta$ aminolevulinic acid to stationary phase, harvested, washed with distilled water. Cells $\left(2.10^{5}\right.$ cells. $\mathrm{ml}^{-}$ $\left.{ }^{1}\right)$ were inoculated in complete medium containing doxycycline as indicated. (A) Optical density reached by the culture was measured 40 hours after the shift. (B) and (C) FAS activity was measured 40 hours after the shift of the corresponding strains. Values are the mean \pm SD of two independent experiments.

\section{Figure 9. Recombinant $\mathrm{Sfh} 2 \mathrm{p}$ inhibits fatty acid synthase activity in vitro.}

Cellular extracts were prepared from strain MRY82-2 (hem1 $14 \mathrm{sh} 2 \Delta$ ) were processed as described in Figure 3 and incubated for 6 hours in $\delta$-aminolevulinic acid and oleate depleted medium. Fatty acid synthase activity was measured one minute after the addition of variable amount of affinity purified recombinant MBP-Sfh2p. Activity is expressed as mU.mg ${ }^{-1}$ protein and is the mean $\pm \mathrm{SD}$ of two independent experiments.

\section{Figure 10. Sfh2p and Fas1p can both localize to lipid particles.}

Yeast strain TDY36-1d (expressing GFP-Sfh2p and Fas1p-3xHA) was grown to stationary phase in the presence of $\delta$-aminolevulinic acid $(\mathbf{A}, \mathbf{B})$ and processed as described in Experimental, or further inoculated and cultivated for 6 hours in complete medium in the absence of oleic acid supplementation $(\mathbf{C})$, or in complete medium containing $\delta$-aminolevulinic acid (D). Separate images of GFP fluorescence (left panel, A), or immunodecorated Fas1p-3xHA (left panel B, C and D) and Nile red fluorescence (middle panel $\mathbf{A}, \mathbf{B}, \mathbf{C}$ and $\mathbf{D}$ ) were captured from the same set of cells. No fluorescence bleed was detected between each channel. The right panel represents transmission images of the cells. 


\section{Table legends}

Table 1. Yeast strains, genotype and source

Table 2. Percentage of SFA following a shift to $\delta$-aminolevulinic and oleic acid depleted medium of hem1A and hem1A sfh2A.

Yeast strains MRY72 (hem1A) and MRY82-2 (hem14sfh2A) were grown to stationary phase in the presence of $\delta$-aminolevulinic acid, harvested, and inoculated in complete medium lacking $\delta$ aminolevulinic and oleic acid. Phospholipids and neutral lipids were extracted from the cells immediately after the shift $(\mathrm{T}=0 \mathrm{~h})$ or 16 hours after the shift $(\mathrm{T}=16 \mathrm{~h})$. The percentage of saturated fatty acids versus unsaturated fatty acids of each lipid species was obtained as described in Experimental procedures. Values are the means \pm SD of at least two independent experiments. 
TABLE 1.

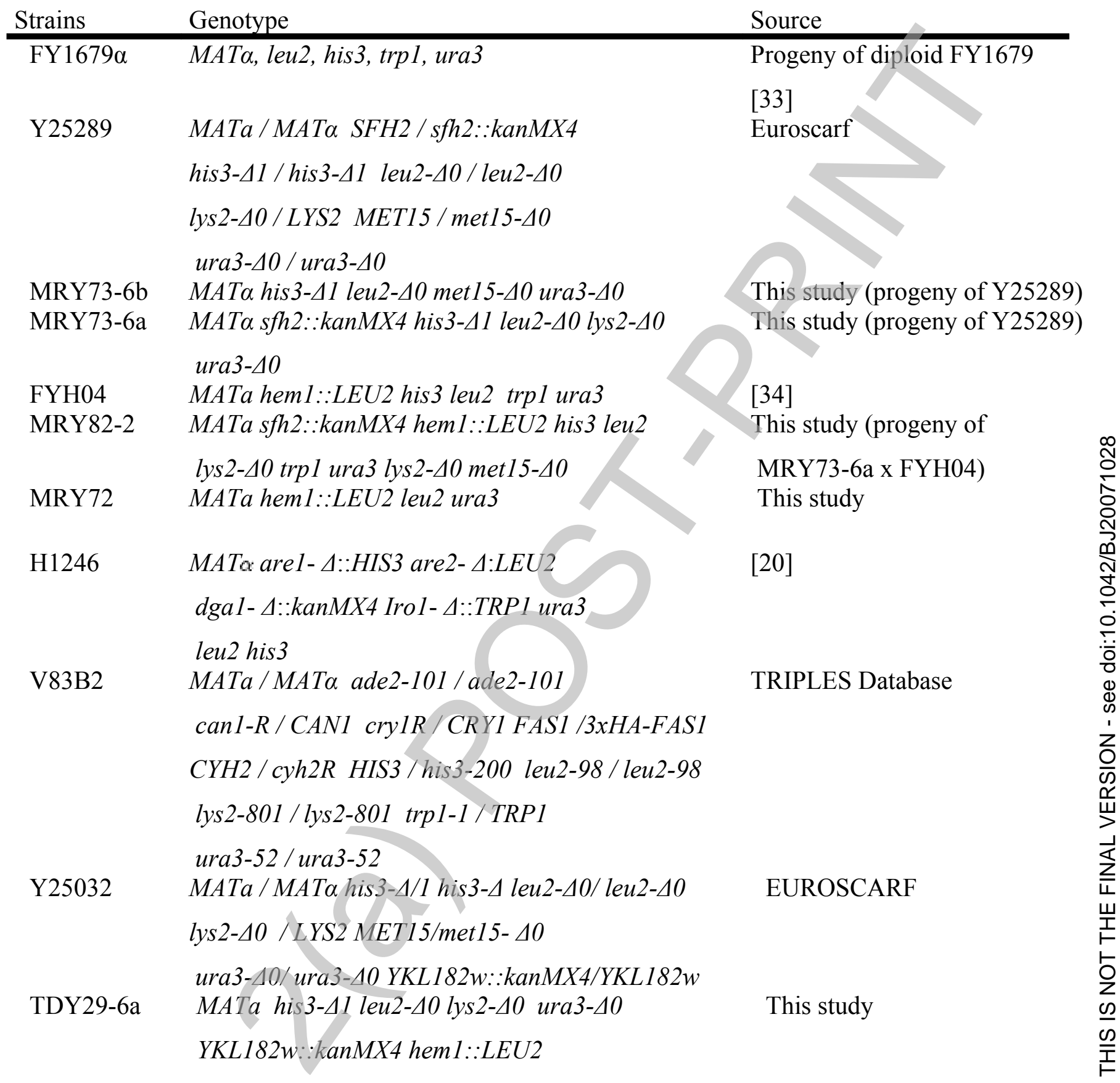

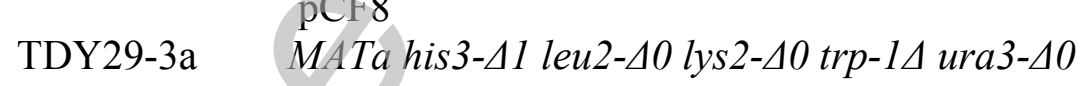

This study

YKL182w::kanMX4 sfh2::KanMX4 hem $1:: L E U 2$

pCF 8

V83B2-d MATa leu2-98 ade2-101 ura3-52 lys2-801

trp1-1 3xHA-FAS1

TDY33-3a MATa leu2 ade2 ura3 lys2 his3 trp1 hem 1::LEU2

$3 x H A-F A S 1$

TDY33-2d MATa leu2 ura3 lys2 his3 trp1 hem1::LEU2

This study (progeny of V83B2)

This study (progeny of MRY82-2 x V83B2-d)

This study (progeny of 
Biochemical Journal Immediate Publication. Published on 6 Sep 2007 as manuscript BJ20071028

\begin{tabular}{|c|c|c|}
\hline & $3 x H A-F A S 1$ sfh $2:$ kanMX4 & MRY82-2 x V83B2-d) \\
\hline TDY36-1d & MATa his 3 leu2 lys 2 ura3 trp 1 sfh $2:: k a n M X 4$ & This study progeny of \\
\hline TDY23-e6 & $\begin{array}{l}3 x H A-F A S 1 \text { hem } 1:: L E U 2 \text { pCG4GFP } \\
\text { MATa hem } 1:: L E U 2 \text { are } 1-\triangle:: H I S 3 \text { are } 2-\triangle:: L E U 2\end{array}$ & $\begin{array}{l}\text { YPCG4-G9d x TDY33-2d } \\
\text { This study (progeny of }\end{array}$ \\
\hline & dgal- $4:: k a n M X 4$ Irol- $\triangle:: T R P 1$ ura3 & MRY82-2 x H1246) \\
\hline TDY23-c4 & 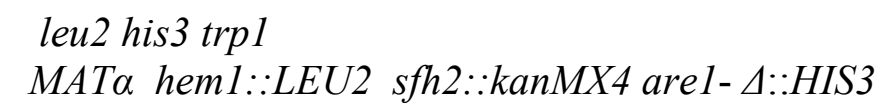 & This study (progeny of \\
\hline & are2- $\Delta:: L E U 2$ dgal- $\triangle:: k a n M X 4$ Iro1- $\triangle:: T R P 1$ & \\
\hline YPCG4_G9d & his3 ura3 trp1 leu2 & H1246) \\
\hline & ura3-40 [pCG4GFP] & \\
\hline
\end{tabular}


TABLE 2.

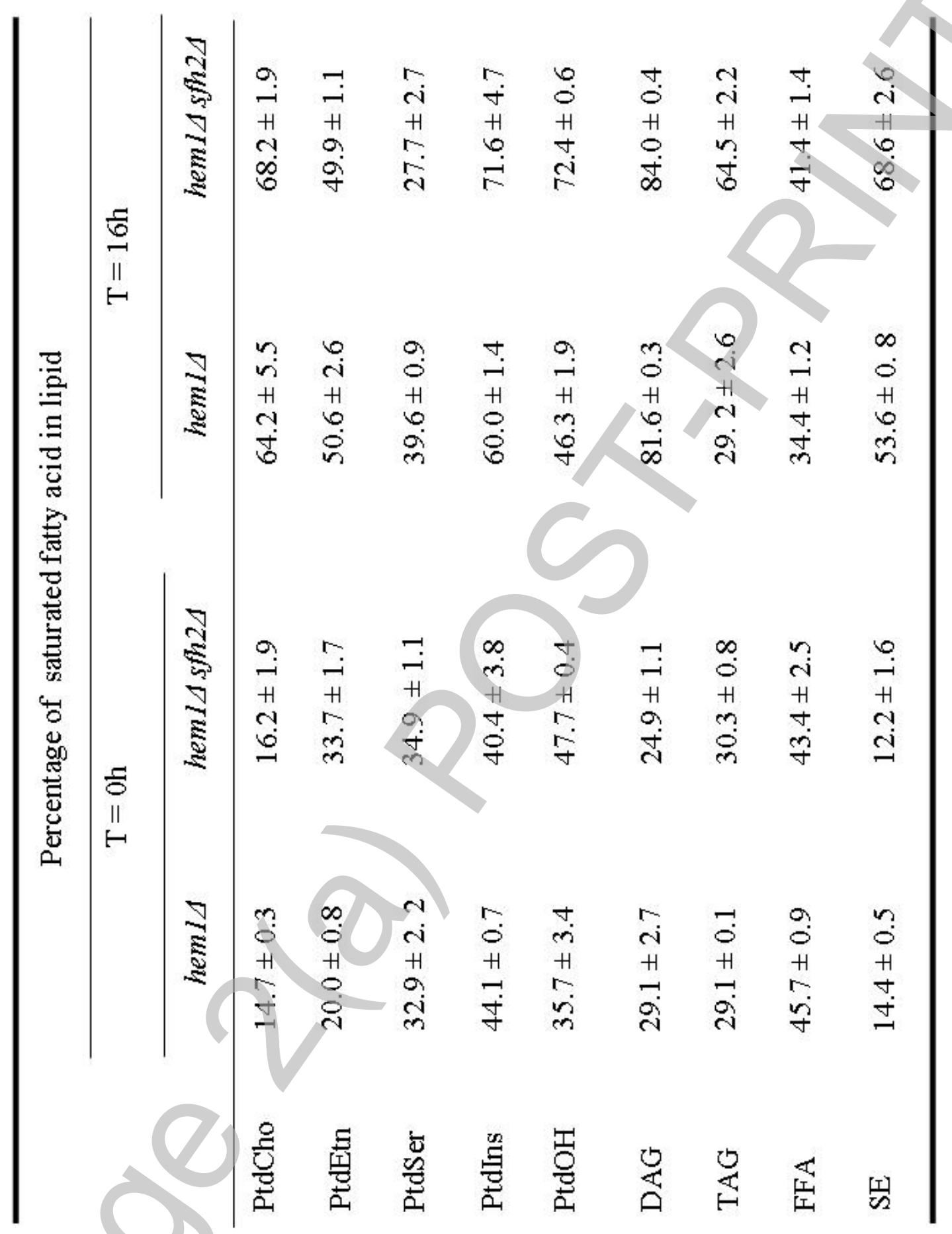


Figure 1

A

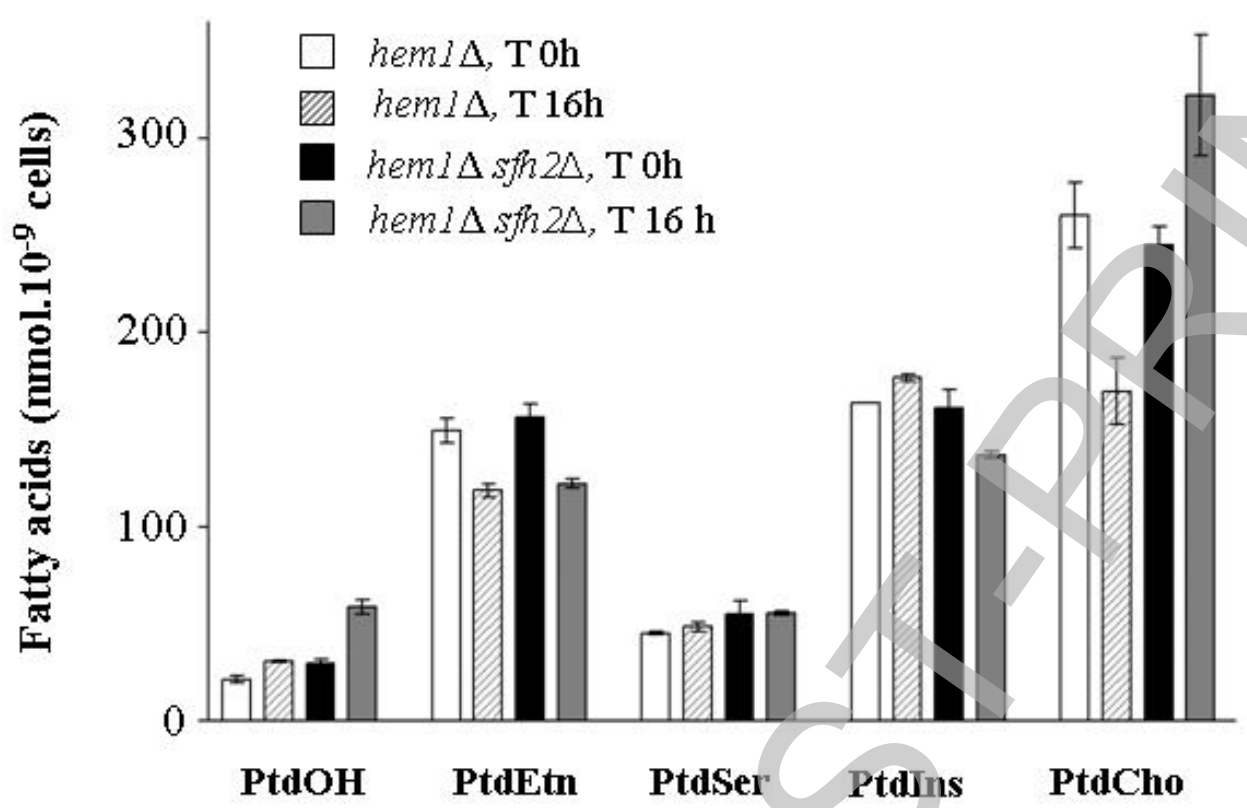

B

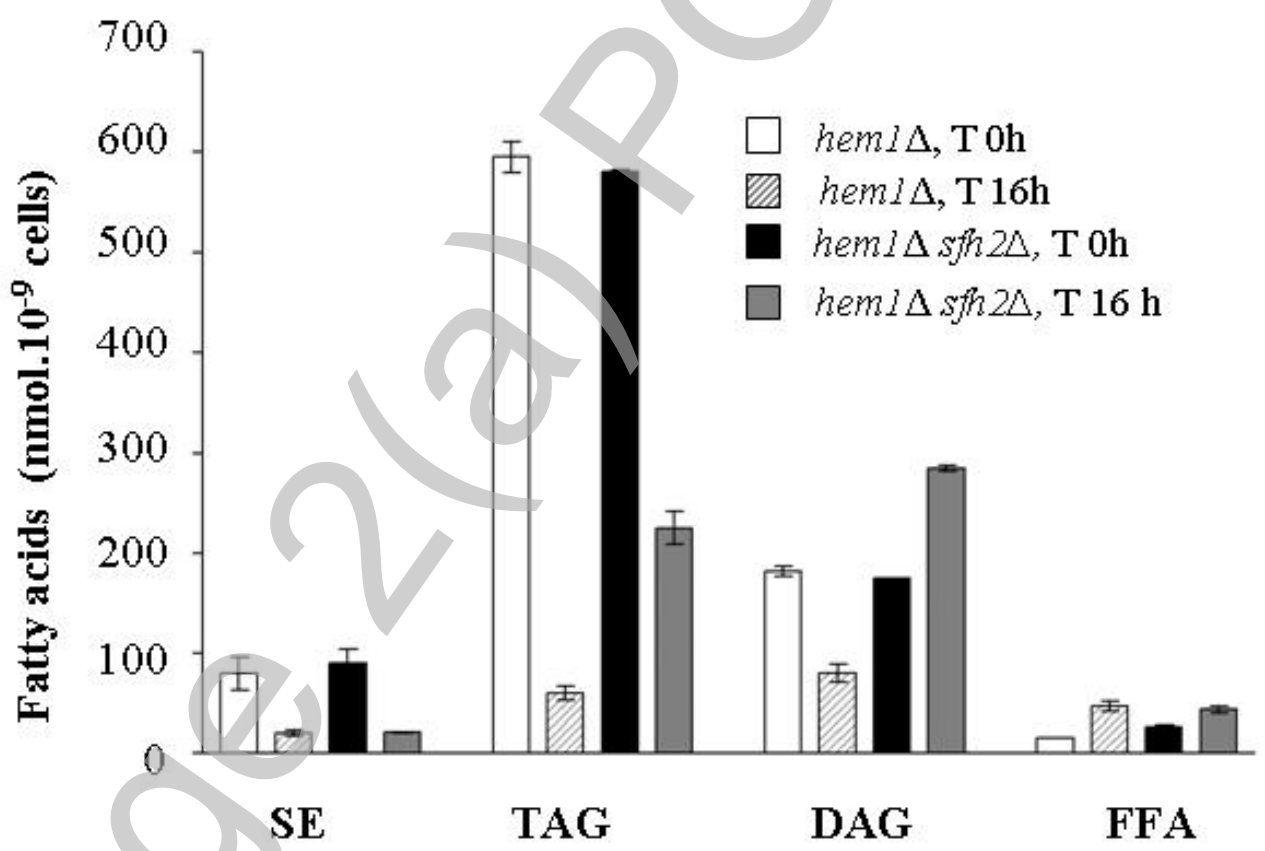


Figure 2
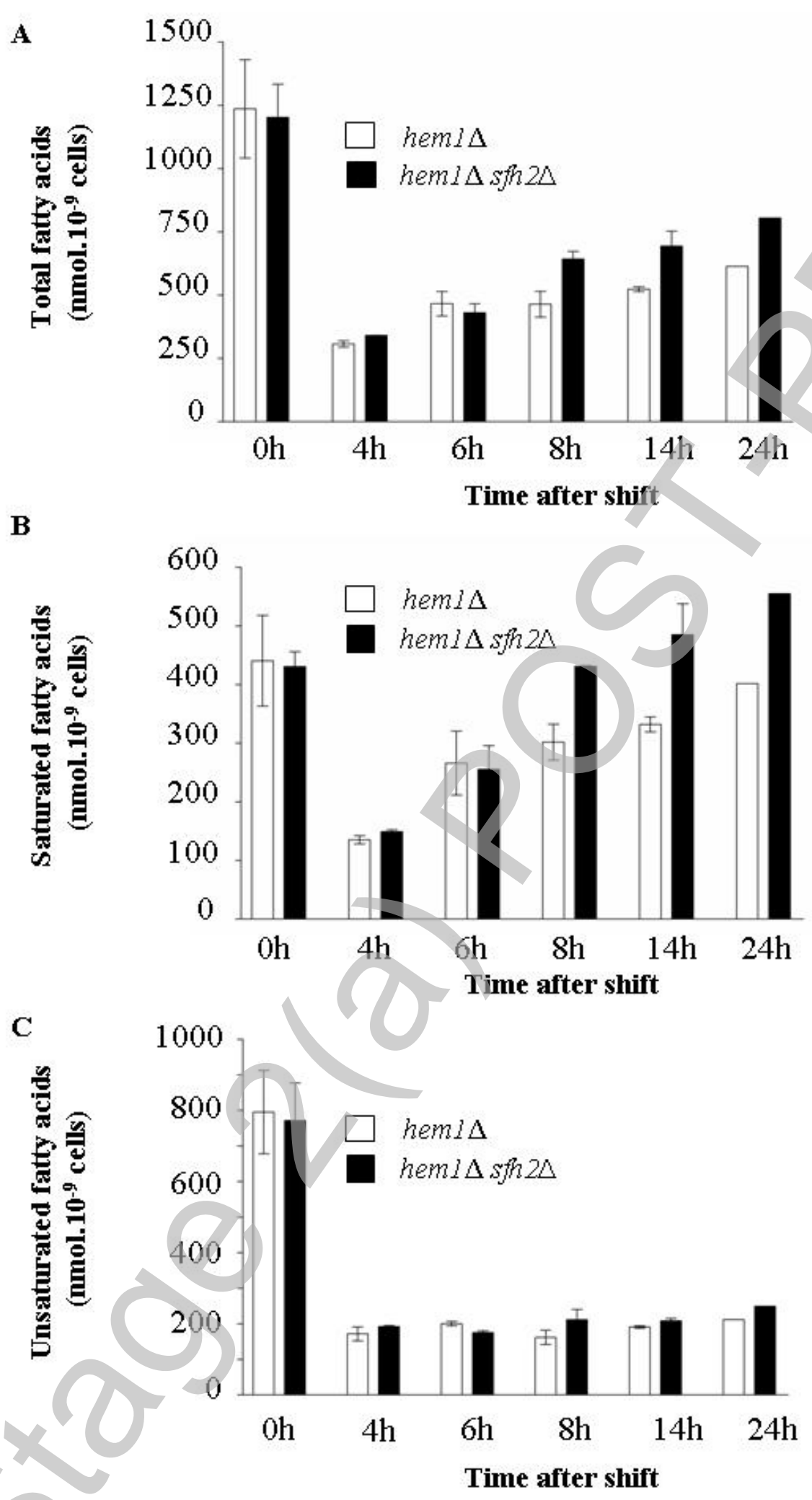
Figure 3

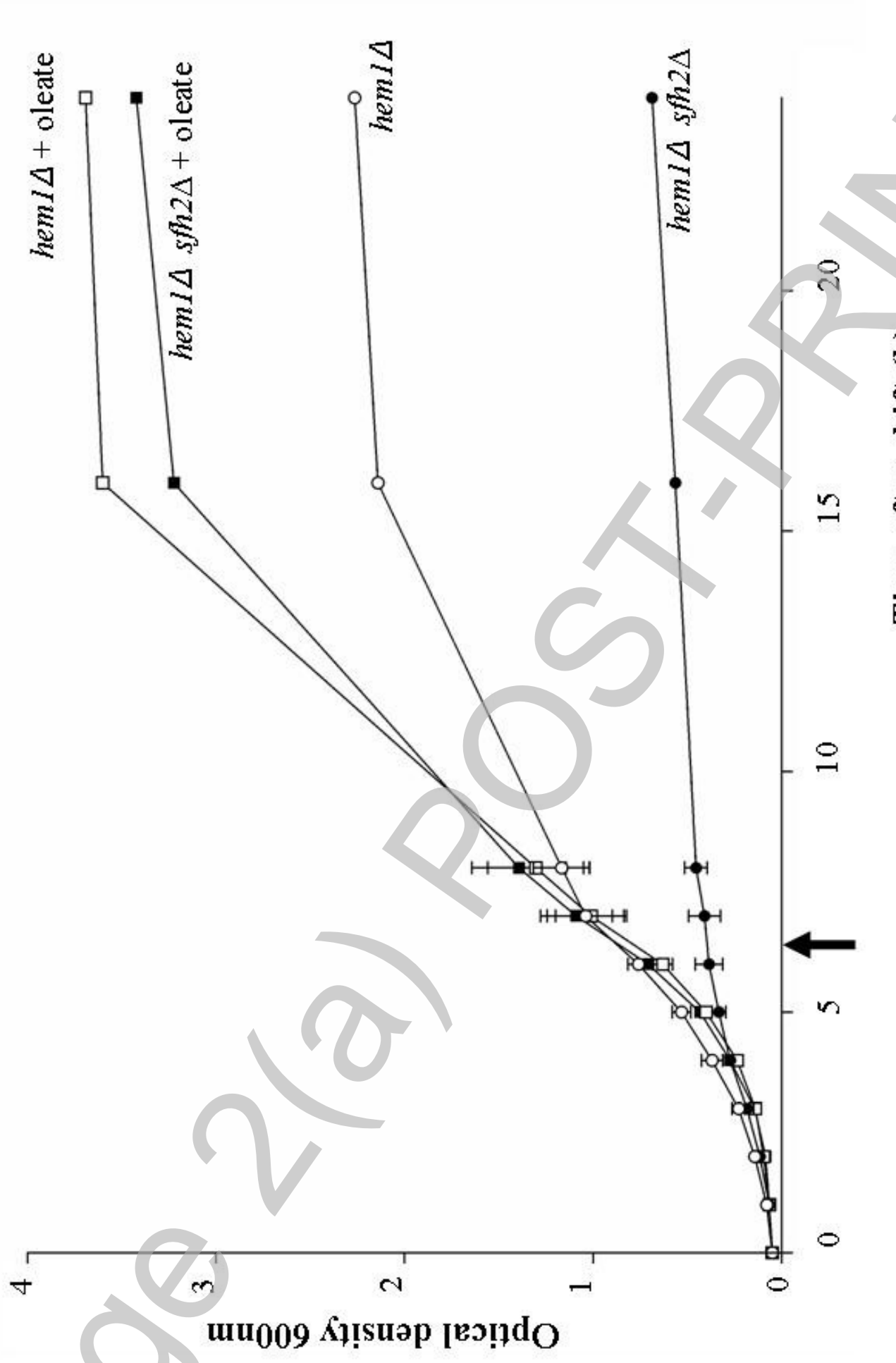


Figure 4 A, B

A

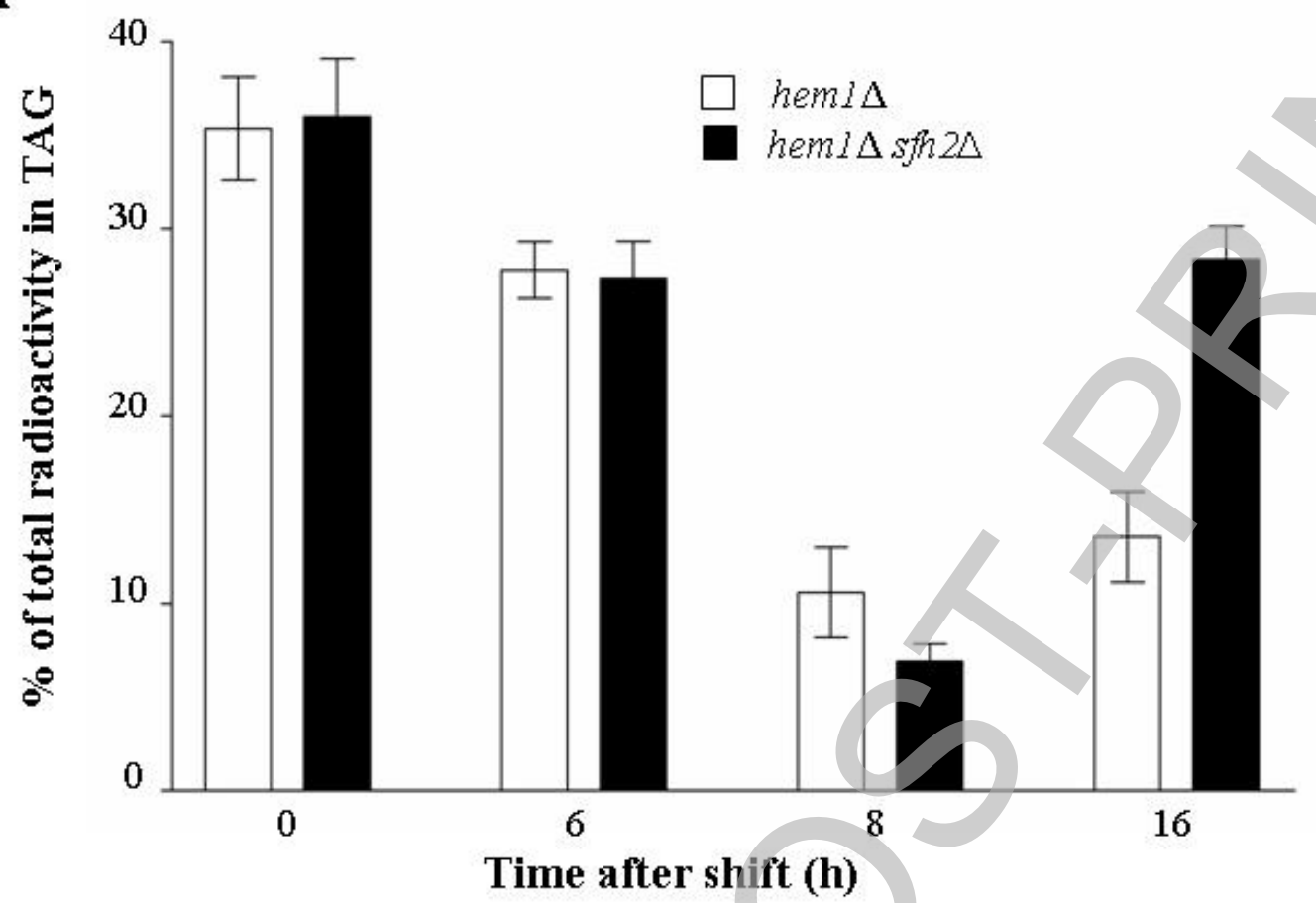

B

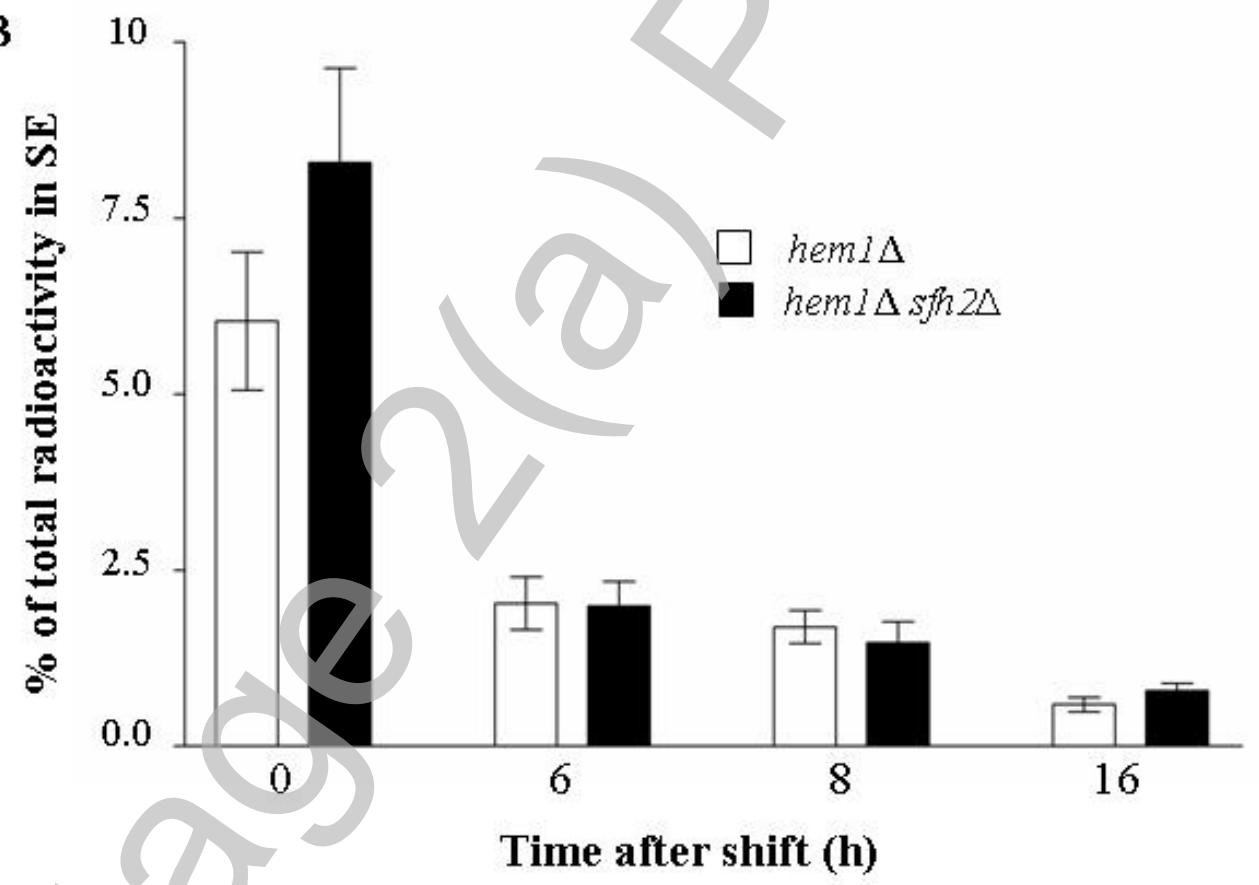

Licenced copy. Copying is not permitted, except with prior permission and as allowed by law. (c) 2007 The Authors Journal compilation (c) 2007 Biochemical Society 
Figure $4 \mathrm{C}$

C

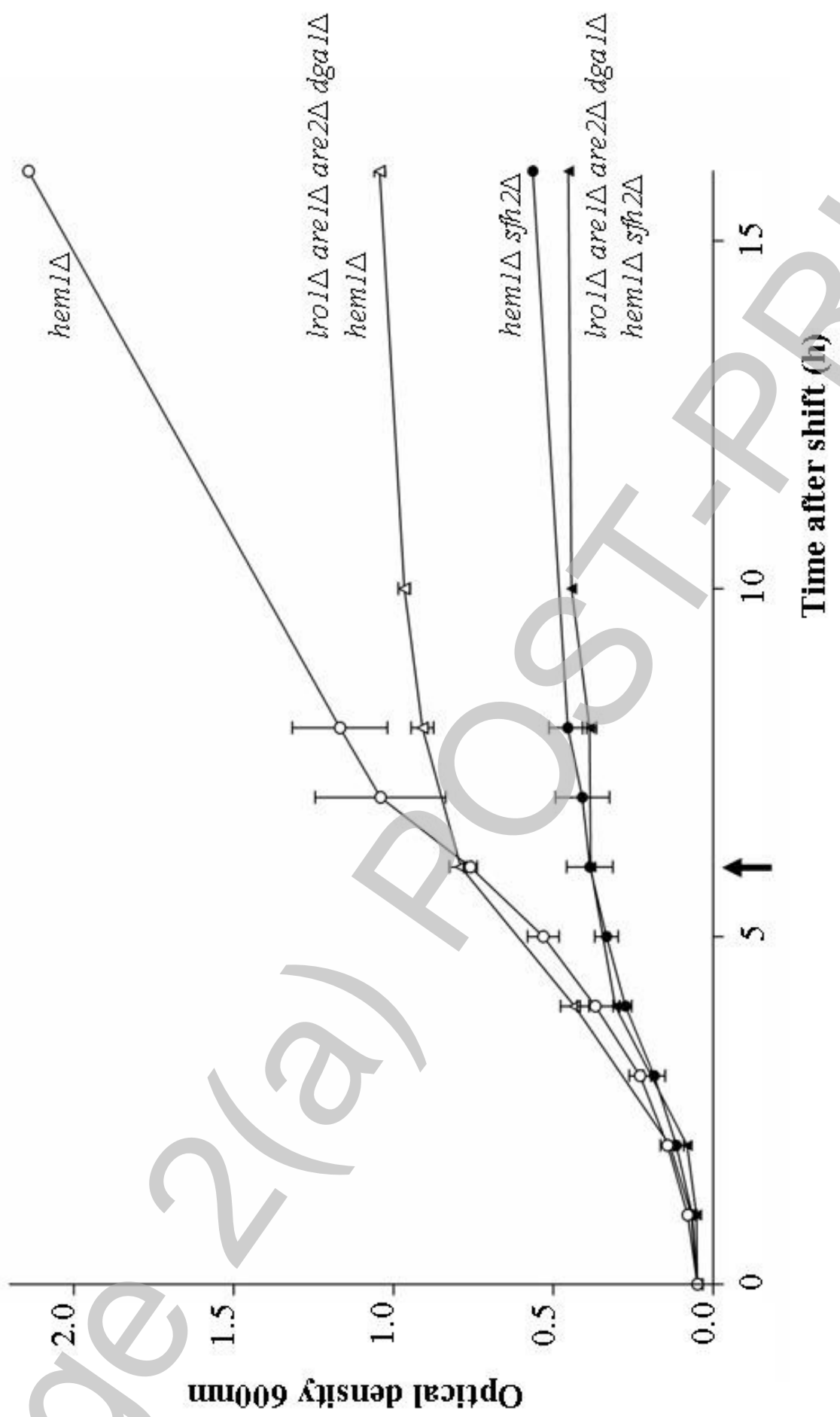


Figure 5

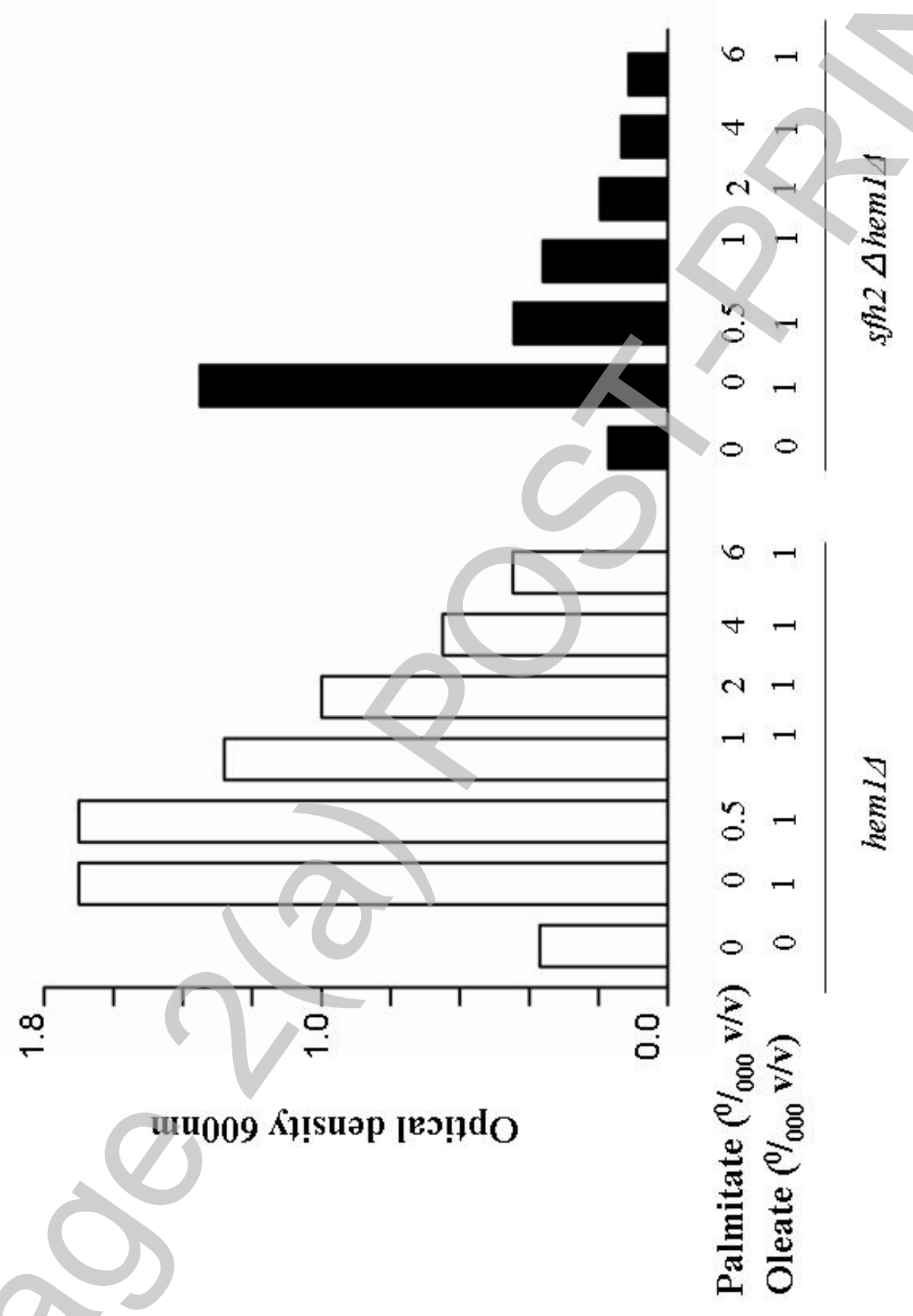


Figure 6

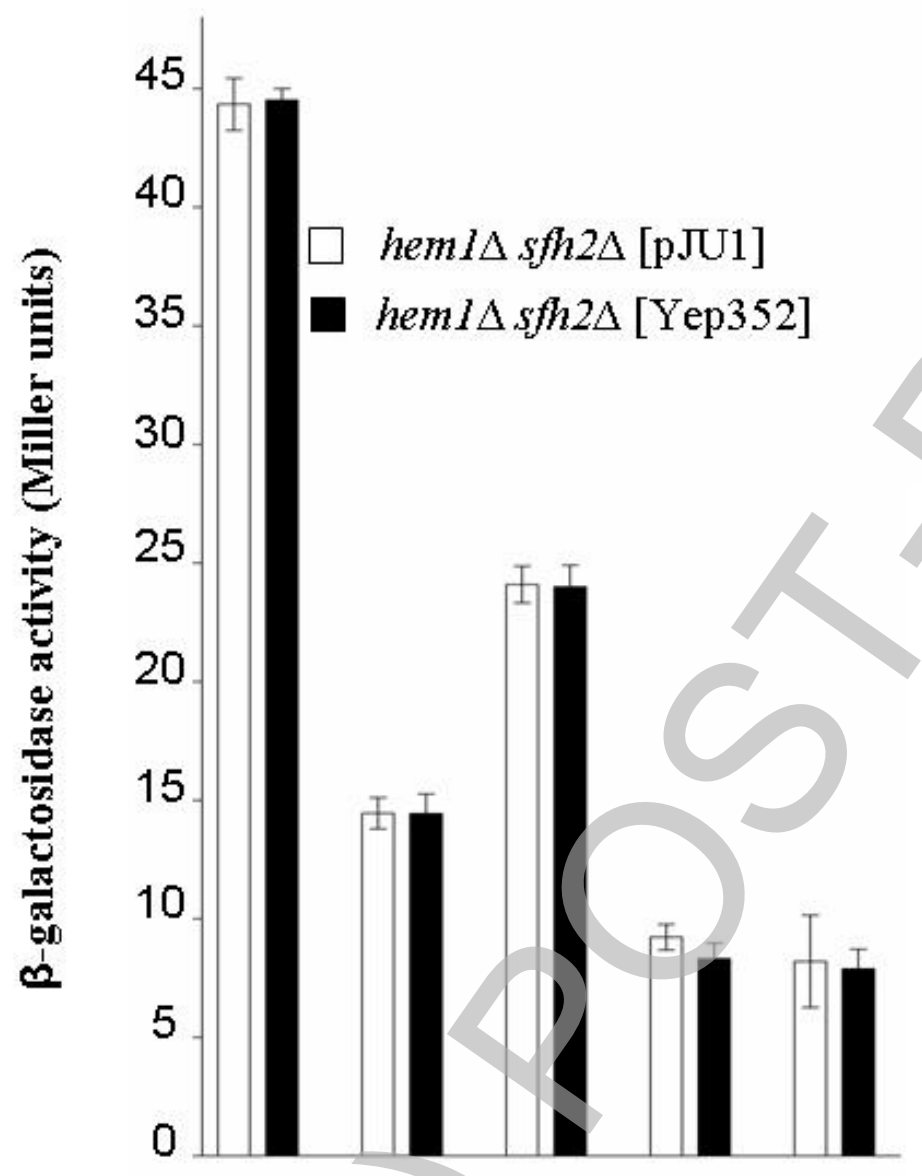

\begin{tabular}{c|c|c|c|c|c|c}
$\delta$-ala & - & - & + & + & + & \\
\hline Oleic acid & - & + & - & + & - & \\
\hline $\begin{array}{l}\text { Palmitic } \\
\text { acid }\end{array}$ & - & - & - & - & + & \\
\hline
\end{tabular}


Figure 7

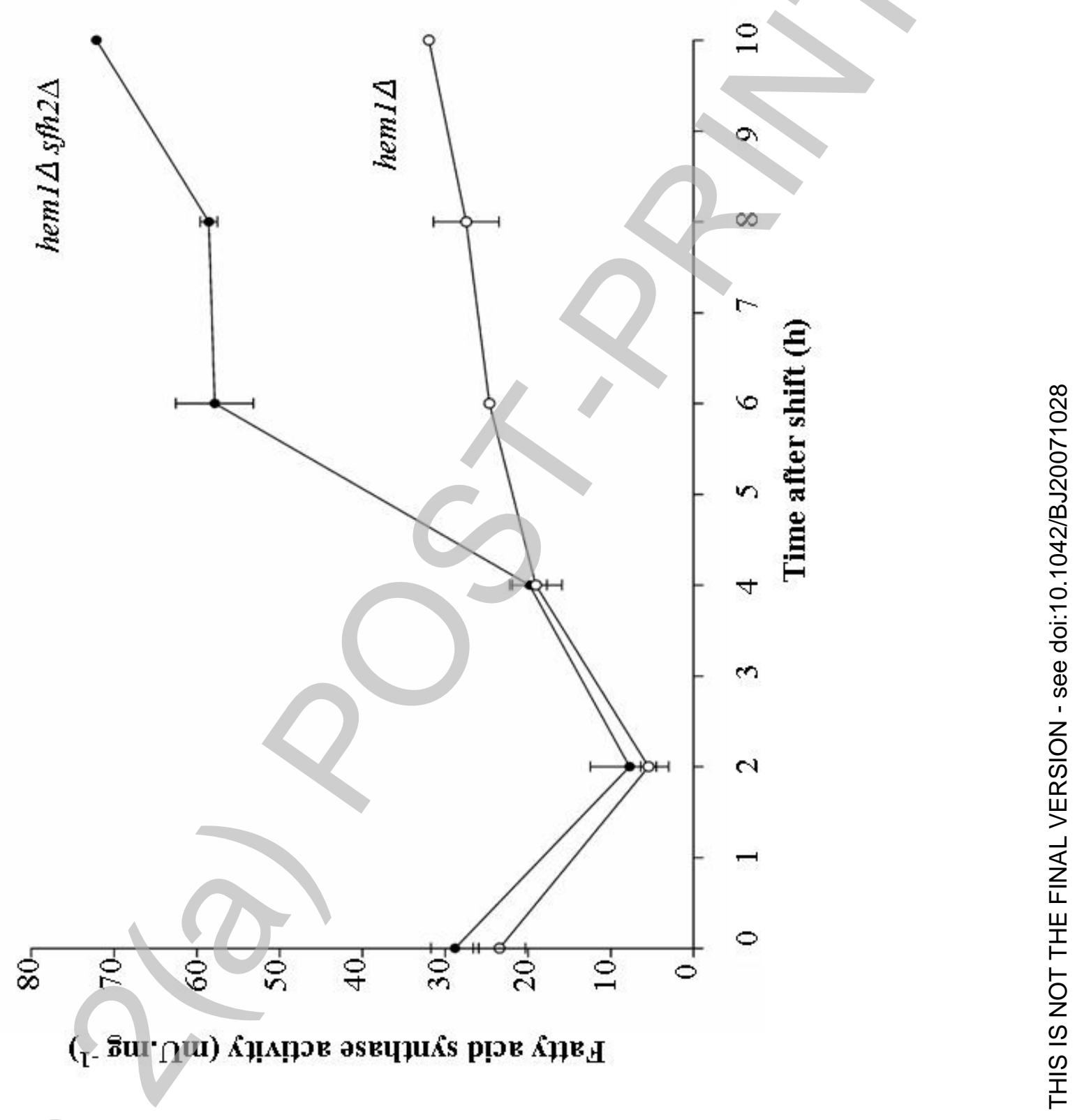


Figure 8

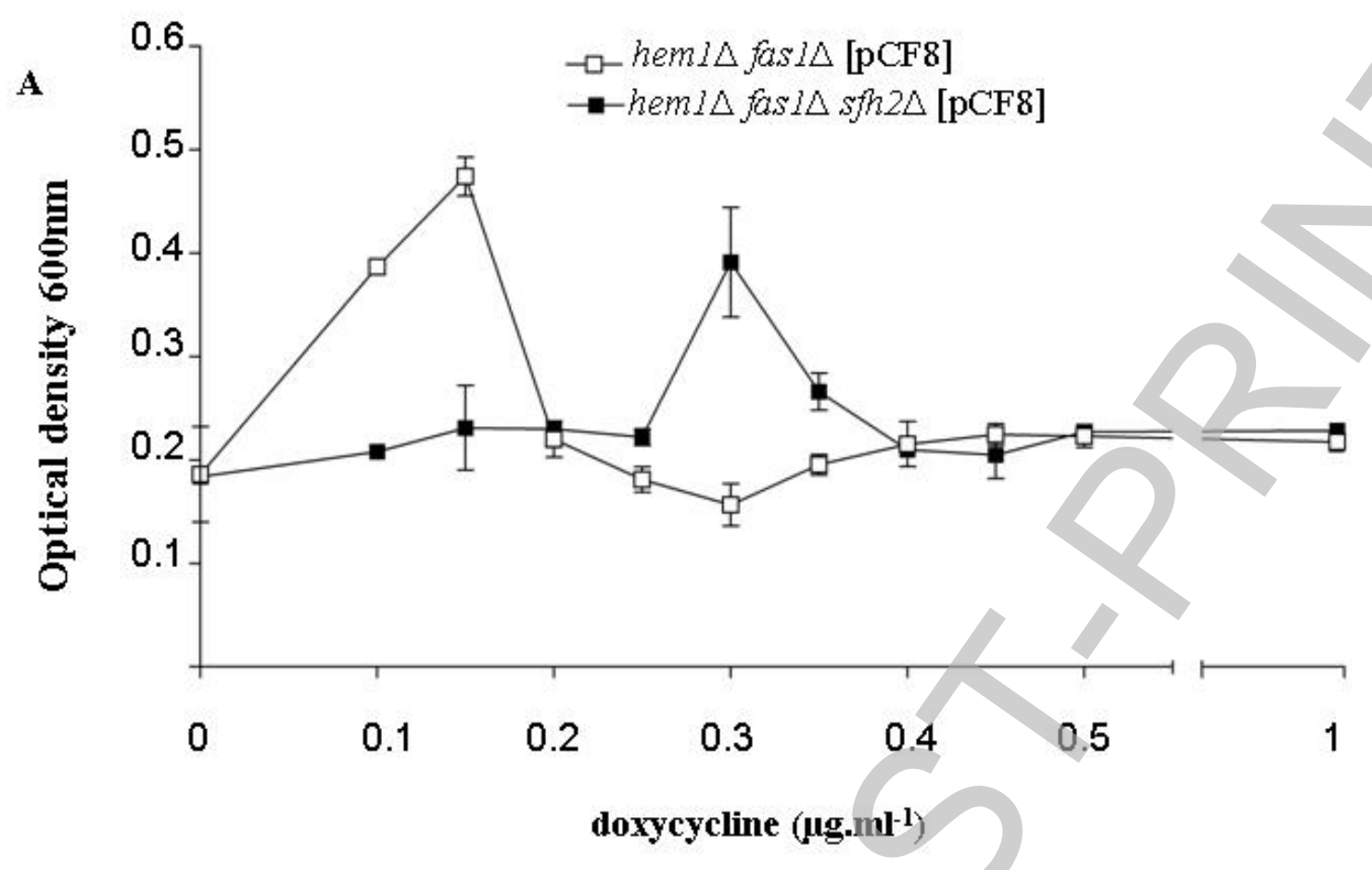

B

C
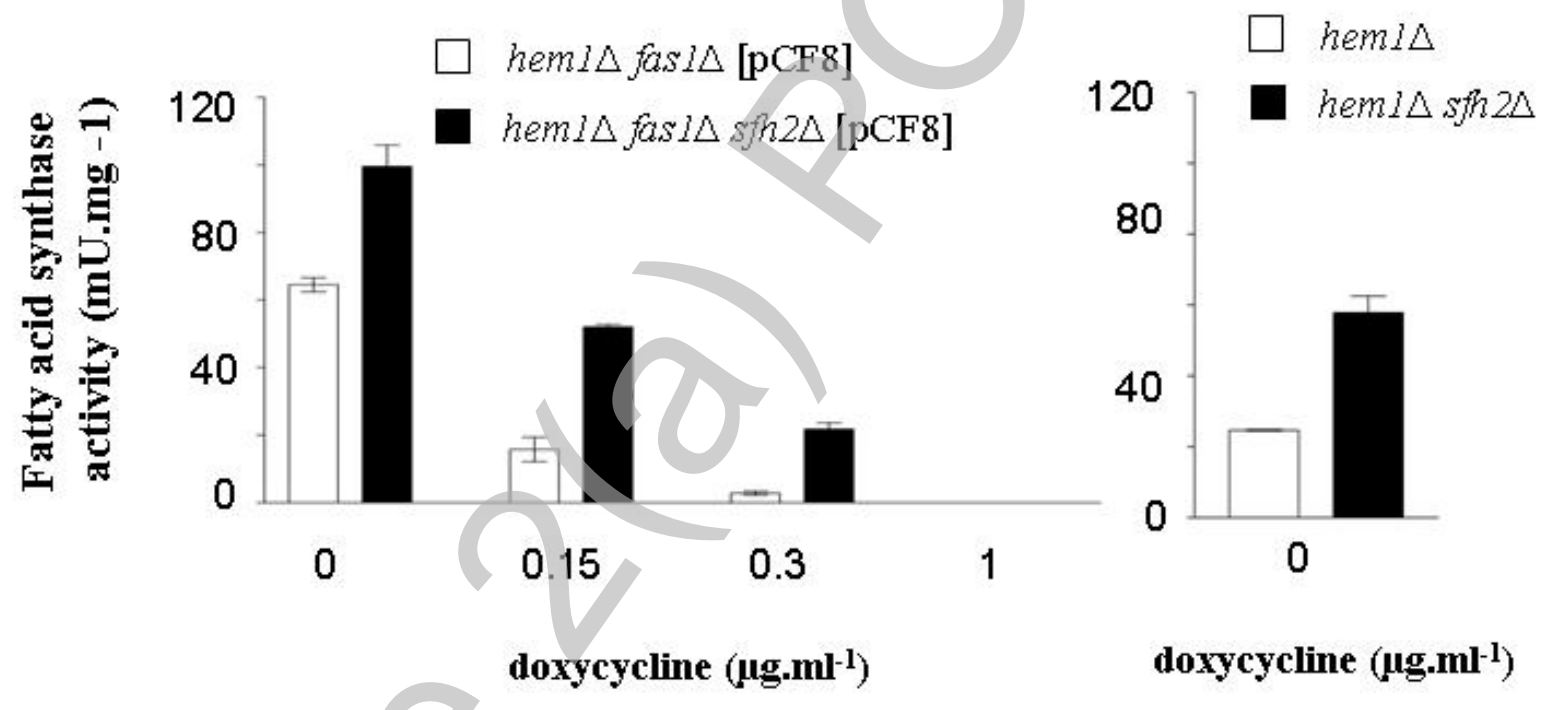

doxycycline ( $\mu$ g.ml- ${ }^{-1}$ ) 
Figure 9

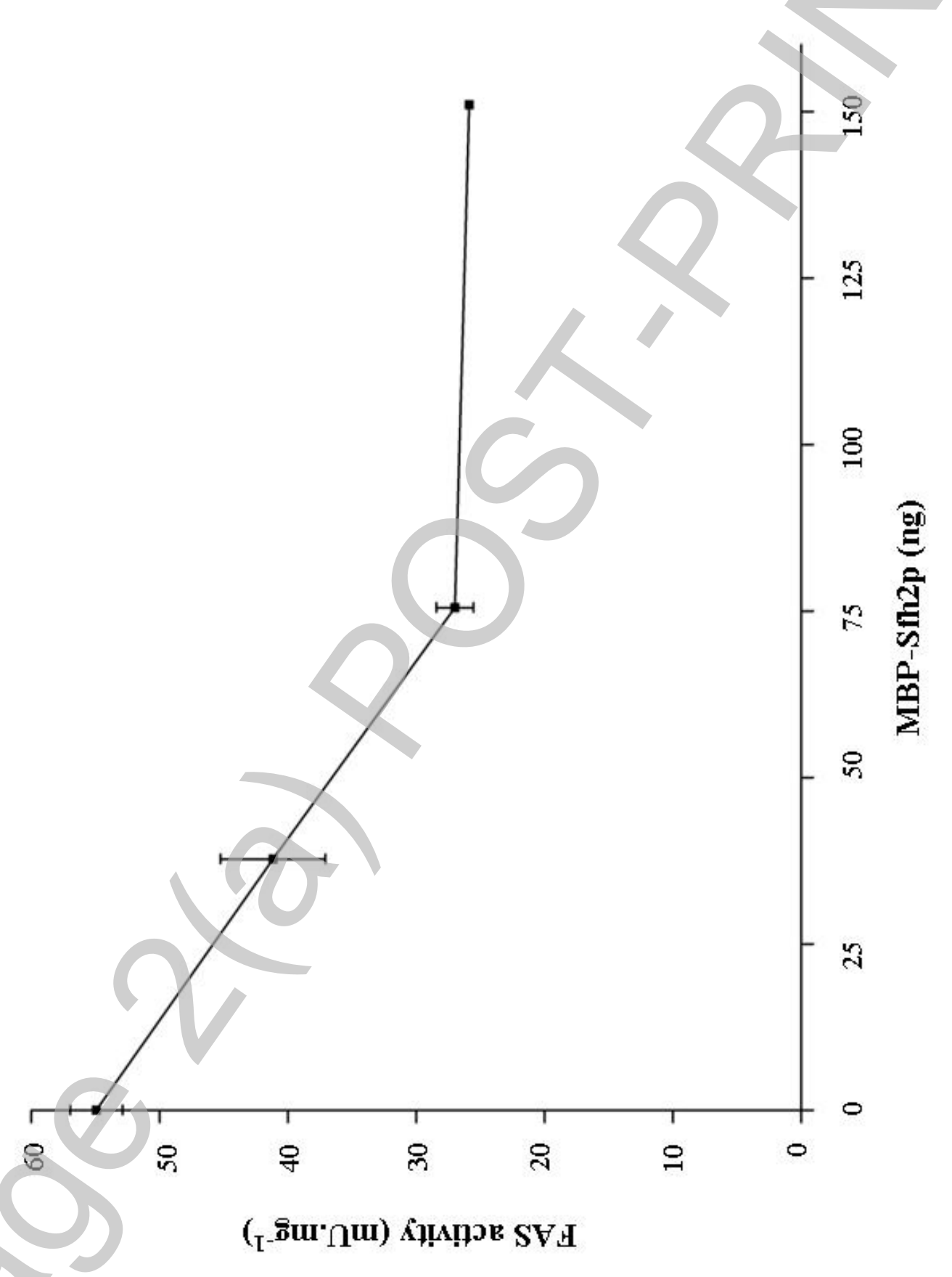


Figure 10
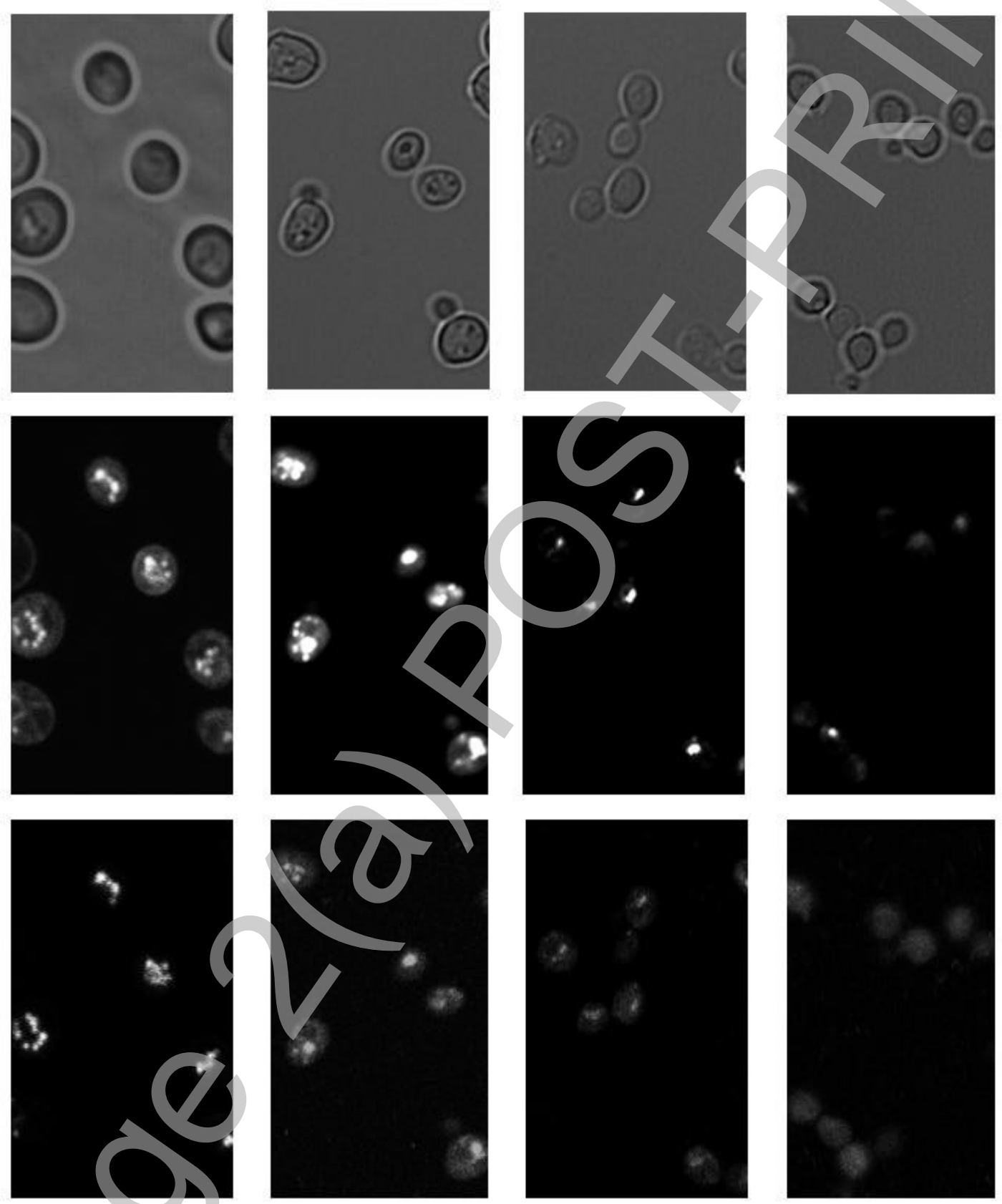

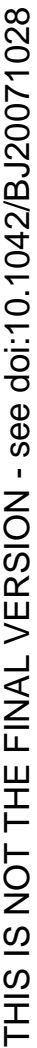

Licenced copy. Copying is not permitted, except with prior permission and as allowed by law. (C) 2007 The Authors Journal compilation (C) 2007 Biochemical Society 